D. Modesto, B. Ye, S. Zlotnik and A. Huerta, "Fast solution of elliptic harbor agitation problems under frequency-direction input spectra by model order reduction and

\title{
Fast solution of elliptic harbor agitation problems under frequency-direction input spectra by model order reduction and NURBS-enhanced FEM
}

\author{
David Modesto ${ }^{1}$, Boyi $\mathrm{Ye}^{2}$, Sergio Zlotnik ${ }^{2}$ and A. Huerta ${ }^{2}$
}

September 12, 2019

\begin{abstract}
Many harbor applications are based on the solution of linear elliptic agitation problems for many spectral conditions. One of the main goals consists in computing the linear combination of numerous simulations of the harbor agitation problem, using monochromatic waves of different spectral components (i.e. frequency and incoming wave direction). In practice, the standard strategy selects the number of wave components according to a prescribed discretization of the $2 \mathrm{D}$ input spectra. The main issue relies on some quantities of interest that are very sensitive to the level of refinement of the spectra, such as the significant wave height at every mesh point or the identification of resonance modes induced by long wave scattering. In many cases, achieving enough quality in these quantities may impose numerous simulations and, consequently, non-practical computer costs. This can drastically limit the final accuracy of results. To overcome this situation, here a new strategy is proposed to efficiently solve a large number of harbor agitation problems derived from dense discretizations of the $2 \mathrm{D}$ input spectra. The strategy is based on the combination of two different numerical approaches. Firstly, each required monochromatic simulation is solved via high order NURBS (non-uniform rational B-splines) enhanced finite elements (NEFEM). More precisely, NEFEM captures the exact harbor geometry using large mesh elements that produce accurate solutions and significant savings on the system size, particularly in long wave cases. Secondly, a model order reduction technique is used to approximate the original elliptic harbor model by a so-called surrogate model. The main advantage is that, once the surrogate model is
\end{abstract}

\footnotetext{
${ }^{1}$ Computer Applications in Science and Engineering, Barcelona Supercomputing Center (BSC-CNS), 08034, Barcelona, Spain.

${ }^{2}$ Laboratori de Càlcul Numèric (LaCàN), ETS de Ingenieros de Caminos, Canales y Puertos, Universitat Politècnica de Catalunya, Barcelona, Spain

Corresponding author: David Modesto. E-mail: david.modesto@bsc.es
} 
constructed, it can be rapidly evaluated to provide simulations for any value of the spectral components within a range of interest, and without the need of solving any new harbor agitation problem (as the standard strategy does). Thus, this enables the possibility of using any desired discretization of the $2 \mathrm{D}$ input spectra with no additional computer cost. The construction of the surrogate model is performed using the proper generalized decomposition method with a novel incremental computation along the frequency dimension. The proposed strategy is discussed, and its superior performance with respect to standard strategies is demonstrated, on two harbor agitation examples with several applications.

Keywords: Model order reduction, Proper Generalized Decomposition, mildslope equation, NEFEM, PML, harbor

\section{Introduction}

Wave propagation in large harbors areas is generally described by linear elliptic depthintegrated models. The usual engineering practice for harbor studies simulates the wave amplification for many random directional incoming waves. Accordingly, the input frequencydirection spectrum of the offshore area is first discretized into a finite number of components. Since model linearity imposes non-interacting solutions of the problem, the harbor agitation can be computed independently for each spectral case. Next, the wave components can be linearly combined a posteriori using the values of the input spectra to generate useful quantities of interest, see for instance [1]. One recurrent application consists in looking for those frequencies generating high wave amplifications for random directional incident waves, and use them to identify potential resonance effects in the harbor [2-4].

The computational cost associated to the production of repetitive simulations for a large number of frequencies, namely $n_{f}$, and incoming wave directions, namely $n_{d}$, may represent a bottleneck for the engineering application. The numerical strategy must provide $n_{f} n_{d}$ solutions for a preselected set of spectral conditions while accounting for sufficient precision to describe the wave field interactions. A brute force approach is used nowadays in the coastal engineering community to deal with this multi parametric scenario, forcing the engineer to decide a priori on the best spectral discretization in terms of the cost/accuracy ratio. This usually either limits the final number of spectral components to be simulated or interpolates solutions to achieve a larger number of them. In the latter case, however, controlling the interpolation error becomes a very difficult task particularly when many parameters are tested. To overcome both limiting issues (i.e. high computational cost and error control) of the standard approach, here an alternative numerical strategy is presented.

The main goal consists in evaluating a so-called surrogate model of the original linear elliptic harbor model using an online-offline model order reduction strategy (MOR) [5,6]. This surrogate model is expressed as an explicit function that generates the solution of the harbor agitation problem not only at any position in the spatial mesh, but also for any 
spectral condition. That is, any combination of frequencies and incoming wave directions within a range of interest. The evaluation of this function is called the online phase of the MOR strategy, and it provides an immediate access to any simulation required by the harbor application of concern. In the other hand, the process of constructing the surrogate model defines the offline phase of the MOR strategy. This phase is computationally intensive, but it requires to be computed only once in a lifetime for a particular harbor geometry and bathymetry. The method applied here is the proper generalized decomposition (PGD) [7,8], with special attention in the construction of surrogate models for both short and long waves. It is a MOR technique that imposes a separable expansion to approximate the elliptic model solution at any spatial point, frequency and incoming wave direction. It has been extensively used in multiple branches of computational mechanics over the last decade, see some examples in [9-11]. The PGD was firstly explored for harbor agitation in [6] but strongly limited to wave periods between $10 \mathrm{~s}$ and $16 \mathrm{~s}$, because a higher frequency range and the non-hermitian properties of the elliptic operator preclude convergence of PGD algorithms [6,12]. In this respect, a novel PGD constructor for short wave cases is also presented in this paper. The new algorithm preserves the convergence of the method in those cases where the standard construction fails. Results demonstrate that solutions ranging from $6 \mathrm{~s}$ of wave period can be effectively obtained with this novel approach on large scale harbor domains.

The PGD rationale is based on a greedy procedure where, in essence, the surrogate model is found by the aggregation of monochromatic solutions of the harbor agitation problem. Thus, the computational cost of the offline MOR phase is directly proportional to the cost of solving the original elliptic model. Aiming to provide an efficient computation of the surrogate model, here an appropriate finite element discretization of the computational domain that generates an optimal number of degrees of freedom (DOF) is also proposed. The idea relies on maximizing the mesh element size while, at the same time, preserving those geometric details that are relevant in the solution of wave propagation problems [13]. Particularly, harbor boundaries which are designed with complex shapes and multitude of corners, see for instance [14]. Within the standard finite element framework this condition may become highly restrictive, because although the design frequencies of the problem may tolerate large elements, their size must be limited by the minimum boundary representation using local mesh refinements [14-16]. Usual harbor agitation examples are encountered in resonance analyses for long wave scattering, see for instance $[15,17]$.

Alternatively, exact geometry-based discretization methods can naturally deal with the small geometric details because they incorporate the exact boundary representation to the discretization of the computational domain. These methods approximate the solution using the same basis that generates the geometry, which normally is a non-uniform rational Bsplines (NURBS) family, instead of classical polynomial basis from standard finite element approaches. However, usual methods like isogeometric analysis [18] propose this treatment for all the elements in the computational mesh, that can lead to important overheads when many simulations for different spectral conditions in the harbor must be simulated. Thus, polynomial basis and FEM methods are still preferable in these cases. 
With this aim, the use of NURBS-enhanced finite elements (NEFEM) [13,19] is proposed here for the first time in harbor agitation problems. NEFEM incorporates the non-uniform rational B-splines description of the boundary to the standard finite element analysis. Thus, only those elements in the mesh with a curved edge on the boundary have a special treatment, and in particular, efficient integration rules are designed to assemble the integral equations of the model on these elements [20]. Since the geometric approach is independent of the mesh size, the spatial discretization can be uniquely controlled by how the solution is approximated (e.g. points per wavelength). This enables the possibility of constructing high order meshes with uniform large elements and without those local refinements usually imposed by the complex harbor geometry. NEFEM has been successfully used in multitude of applications for wave and fluid problems in need of accurate geometric descriptions, see for instance [21,22] and the references therein.

This paper is structured as follows. The elliptic harbor model and its physical and artificial boundary conditions are firstly presented. Secondly, the numerical strategy based on MOR and NEFEM contributions is explained. Next, three different applications for harbor agitation problems are solved to demonstrate the potential advantages of the proposed approach. They include a resonance study based on a literature case in the Marina di Carrara harbor (Italy), and a wave height computation ranging from small to high wave periods in the Barcelona harbor (Spain). And finally, some concluding remarks are done.

\section{Harbor agitation model}

The elliptic wave propagation model based on the well-known mild slope equation (MSE) [23, 24] for slow-varying bathymetries and monochromatic waves is considered. Additionally, the PML technique [25] is applied following the recent implementation for harbor agitation problems presented in [26] to deal with the Sommerfeld radiation condition and produce an efficient truncation of the unbounded domain.

The MSE in a domain $\Omega \in \mathbb{R}^{2}$ with PML and bathymetry $b(x, y) \in \mathbb{R}$ writes

$$
\boldsymbol{\nabla} \cdot\left(c c_{g} \mathbf{P} \boldsymbol{\nabla} \phi\right)+k^{2} c c_{g} s_{x} s_{y} \phi=f \quad \text { in } \Omega,
$$

for all the spatial coordinates $(x, y) \in \Omega$. In $(1), \phi(x, y) \in \mathbb{C}$ denotes the (complex) wave surface elevation, $k(b, \omega) \in \mathbb{R}$ is the wavenumber, $\omega \in \mathbb{R}$ is the angular frequency of the monochromatic incoming wave, $c=\omega / k \in \mathbb{R}$ is the phase velocity and $c_{g}=d \omega / d k \in \mathbb{R}$ is the group velocity. The dispersion relation

$$
\omega^{2}=k g \tanh (k b)
$$

is used to define the group velocity as $c_{g}=g\left[\tanh (k b)+k b \operatorname{sech}^{2}(k b)\right] /(2 \omega)$, with $g$ the acceleration of gravity. If higher bathymetry gradients are of concern, or nonlinear effects are relevant in the wave propagation area, such as wave breaking or bottom friction, the equations (1) and (2) can be properly modified, see for instance [27-29]. 


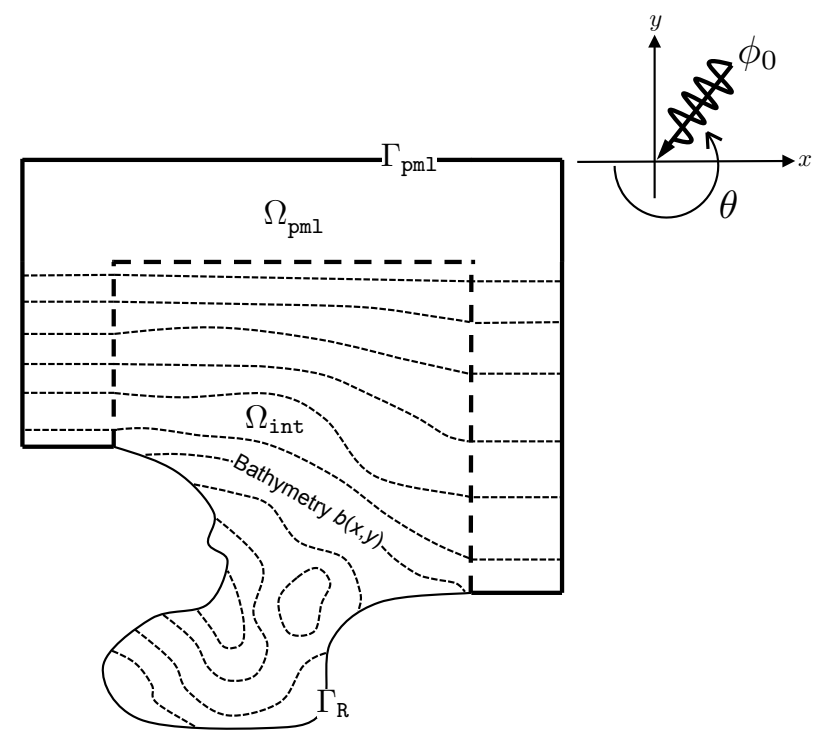

Figure 1: Sketch of the computational domain for harbor agitation problems with PML.

The computational domain $\Omega$ is divided into a region of interest $\Omega_{\text {int }}$, and an artificial PML region $\Omega_{\mathrm{pml}}$, such that $\Omega=\left(\Omega_{\text {int }} \cup \Omega_{\mathrm{pml}}\right) \subset \mathbb{R}^{2}$. The exterior artificial boundary is denoted by $\Gamma_{\mathrm{pml}}$ as depicted in figure 1 . Note that the PML surrounds $\Omega_{\text {int }}$ in order to absorb outgoing waves. To ensure the absorbing properties of the artificial layer, see [30,31], the coefficients $c, c_{g}$ and $k$ in (1) are assumed constant along the normal direction to $\Gamma_{\mathrm{pml}}$ in the PML region. As demonstrated in $[26,32]$ this only implies a reasonable simplification of the bathymetry in the exterior harbor area, see figure 1.

The source term in (1) accounts to absorb only the scattered waves in the PML region, namely

$$
f= \begin{cases}0 & \text { if }(x, y) \in \Omega_{\text {int }}, \\ \nabla \cdot\left(c c_{g} \mathbf{P} \boldsymbol{\nabla} \phi_{0}\right)+k^{2} c c_{g} s_{x} s_{y} \phi_{0} & \text { if }(x, y) \in \Omega_{\mathrm{pml}},\end{cases}
$$

where $\phi_{0} \in \mathbb{C}$ denotes the directional incident wave. The diagonal anisotropy matrix $\mathbf{P}$ defines the absorption in the PML medium

$$
\mathbf{P}=\left(\begin{array}{cc}
s_{y} / s_{x} & 0 \\
0 & s_{x} / s_{y}
\end{array}\right)
$$

where $s_{x}=1+\sigma_{x} / \omega$ and $s_{y}=1+\sigma_{y} / \omega$ are the absorption parameters along the two cartesian directions. The choice of the absorbing functions $\sigma_{x}(x) \geq 0$ and $\sigma_{y}(y) \geq 0$, which vanish in $\Omega_{\text {int }}$ to keep the interior solution unperturbed by the artificial layer, determines the absorption rate inside the PML. Usually, these functions are defined as monotonic polynomials along the corresponding cartesian absorbing direction [26, 33, 34].

Boundary condition supporting equation (1) on reflecting/absorbing boundaries, namely $\Gamma_{\mathrm{R}}$, is given by

$$
\boldsymbol{n} \cdot\left(c c_{g} \mathbf{P} \boldsymbol{\nabla} \phi\right)-i k c c_{g} \alpha \phi=0 \quad \text { on } \Gamma_{\mathrm{R}}
$$


where $i=\sqrt{-1}$ is the imaginary unit, $\boldsymbol{n}$ is the outer unit normal, and $\alpha \in[0,1]$ is a reflection/absorption experimental parameter controlling the properties of the boundary (i.e. $\alpha=0$ yields to total reflection and $\alpha=1$ produces perfect absorption). Note in figure 1 that $\Gamma_{\mathrm{R}}$ is always included as a boundary of the interior domain $\Omega_{\text {int }}$, where the PML matrix $\mathbf{P}$ becomes the identity matrix, and thus equation (5) coincides with the classical condition derived in [35].

On the other hand, a first order non-reflecting boundary condition is imposed on the outer artificial boundary $\Gamma_{\mathrm{pm} 1}$, that is

$$
\boldsymbol{n} \cdot\left(c c_{g} \mathbf{P} \boldsymbol{\nabla} \phi\right)-i k c c_{g} \phi=\boldsymbol{n} \cdot\left(c c_{g} \mathbf{P} \boldsymbol{\nabla} \phi_{0}\right)-i k c c_{g} \phi_{0} \quad \text { on } \Gamma_{\mathrm{pml}},
$$

which tries to minimize those possible spurious reflections that have not been absorbed by the PML. Summarizing, equation (1) with boundary conditions (5) and (6) define the elliptic harbor agitation model, to be solved provided the frequency $\omega$ and the directional incident wave $\phi_{0}$ for fixed bathymetry.

\subsection{Variational form of the elliptic model}

The high order finite element method is intensively used when solving elliptic models. This is mainly based on their mesh adaptivity to complex harbor geometries, in addition to control of wave dispersion errors when high frequencies are of concern (i.e. the error between numerical and exact wavelength), see [36]. Since the numerical techniques further presented in this paper also work on the finite element framework, some standard notations must be briefly formalized first.

Let us write the variational or weak form of the MSE model as

$$
a(\phi, v)=l(v) \text { for all } v(x, y),
$$

that yields from integration by parts of the weighted residual of (1). In equation (7a), the bilinear and linear functionals, $a(\cdot, \cdot)$ and $l(\cdot)$ respectively, are defined as

$$
\begin{aligned}
a(\phi, v)=\left(k^{2} c c_{g} s_{x} s_{y} \phi, v\right)_{\Omega}-\left(c c_{g} \mathbf{P} \nabla \phi, \nabla v\right)_{\Omega} & \\
& +i \alpha\left\langle k c c_{g} \phi, v\right\rangle_{\Gamma_{\mathrm{R}}}+i\left\langle k c c_{g} \phi, v\right\rangle_{\Gamma_{\mathrm{pm} 1}}
\end{aligned}
$$

and

$$
l(v)=(f, v)_{\Omega}+\left\langle\boldsymbol{n} \cdot\left(c c_{g} \mathbf{P} \nabla \phi_{0}\right)-i k c c_{g} \phi_{0}, v\right\rangle_{\Gamma_{\mathrm{pm}}} .
$$

In the previous and following equations, standard definitions are used for the inner product $(\phi, v)_{\mathcal{D}}=\int_{\mathcal{D}} \phi \bar{v} d \mathcal{D}$ of complex functions in any domain $\mathcal{D}$, and product $\langle\phi, v\rangle_{\partial \mathcal{D}}=$ $\int_{\partial \mathcal{D}} \phi \bar{v} d \Gamma$ of the traces over $\mathcal{D}$, where $\bar{v}$ denotes the complex conjugate of $v$. 


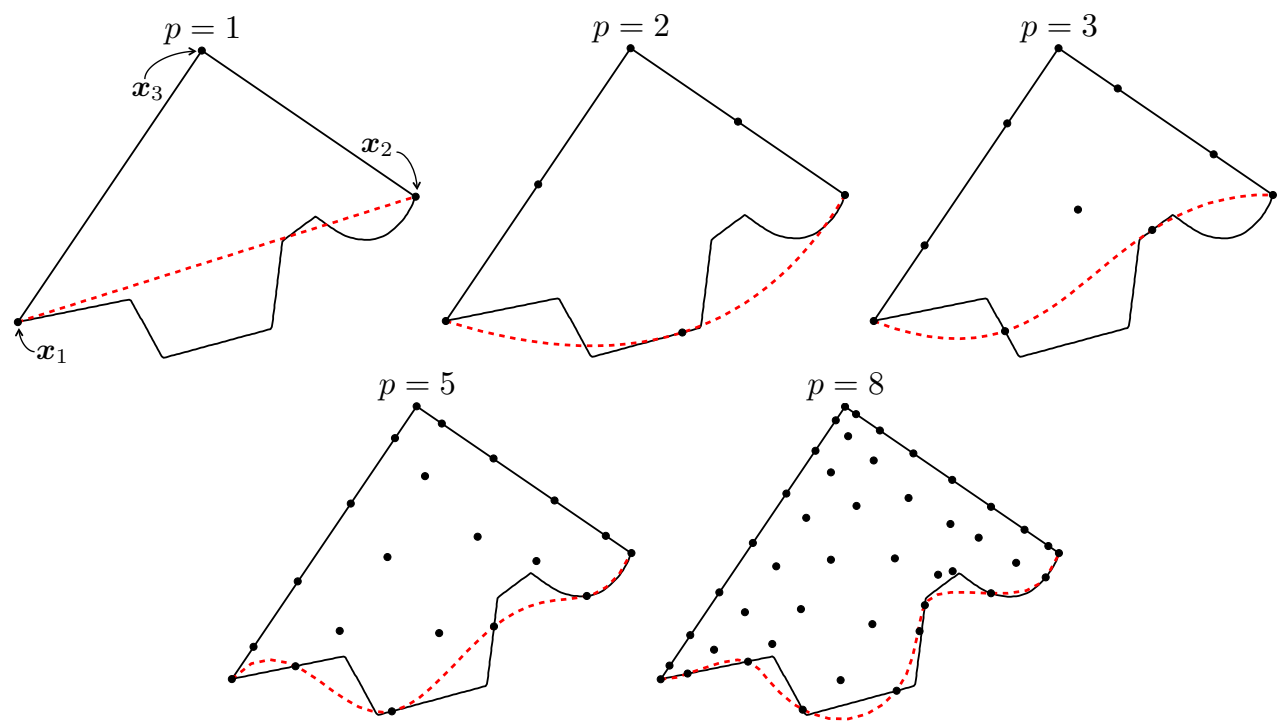

Figure 2: Boundary representation (dashed red line) using FEM for different interpolation order $p$. Coordinates $\boldsymbol{x}_{i}=\left(x_{i}, y_{i}\right)$ of each $i$-th element vertex are indicated. The edge between vertices $\boldsymbol{x}_{1}$ and $\boldsymbol{x}_{2}$ (black solid line) represents the exact boundary.

\section{$3 \quad$ Numerical strategy}

In this section, some advanced numerical techniques are proposed to generate very fast solutions of (1) for any discretization of the 2D input spectra, i.e. any combination of frequencies and incoming wave directions, while providing enough accuracy in outputs of interest. The strategy combines two different approaches presented next.

\subsection{NURBS-enhanced finite elements (NEFEM)}

First, the case of efficiently solving the harbor agitation problem for monochromatic waves (i.e. only one spectral component) is briefly considered here. This is the baseline case controlling the computational cost of the MOR strategy detailed in further sections. Therefore, deriving finite element meshes that induce reduced linear systems is a crucial aspect. Note, moreover, that the use of any numerical technique ensuring this condition is not exclusively limited to the MOR case, but it can be useful for any application requiring numerous solutions of (1).

The basic idea to increase the performance when solving one simulation of the harbor agitation problem relies in using very large high order elements in the mesh, aiming to reduce the total number of degrees of freedom (DOF). Unfortunately, standard finite element methods fail in providing sufficient accuracy in the solution under these large discretizations. More precisely, they are unable to capture the harbor geometry in presence of numerous small geometric details, that include corners, dikes and complex docks, among 
many others. Figure 2 illustrates this case with an example of large element having an edge on a boundary with complex shape. Note how the exact geometry of the boundary is not properly represented using standard high order FEM even with 8th order approximations, which certainly are above the maximum order used in practice. This difference between approximated and exact boundary degrades the accuracy of solutions in scattering problems (e.g. the MSE model) because they are very sensitive to the small geometric details [13].

To overcome this situation, here the NURBS-enhanced finite element method (NEFEM) is applied. NEFEM proposes a particular treatment only for those elements having a curved edge on the boundary of the domain. In particular only the exact description of this curved edge is incorporated to the method, while the piecewise polynomial approximation of the solution is retained from the FEM approach. The rest of elements in the mesh follow a standard FEM implementation preserving all the properties of the method (e.g. convergence, error estimators,...). Thus, NEFEM efficiently combines the advantages of exact-geometry based approaches and the efficiency provided by the polynomial basis for the major part of the computational domain. Results of section 4 demonstrate that NEFEM can significantly reduce the final number of DOF compared to standard high order FEM when solving harbor agitation problems for fixed frequency and incoming wave direction.

For clarity purposes, the reader is addressed to $[13,20,37]$ for the fundamental details of NEFEM. In summary, embedding NEFEM functionalities for boundary elements into existing FEM codes does not demand high implementation efforts. The only modification resides in the computation of boundary integrals present in the weak equations (7) for curved boundaries. Moreover, some algorithms have been designed to generate automatic NEFEM meshes, see for instance [38].

\subsection{Proper generalized decomposition solver}

This section describes the online-offline MOR procedure that provides very fast solutions of the MSE in cases where numerous spectral components must be analyzed. In particular, let us formalize that all the frequencies of interest are included in the $1 \mathrm{D}$ domain $I_{\omega} \subset \mathbb{R}$, and all the incoming wave directions, generated by an incoming wave angle $\theta$ as depicted in figure 1 , are included in the $1 \mathrm{D}$ domain $I_{\theta} \subset \mathbb{R}$. The goal here is to find an explicit function $\phi(x, y, \omega, \theta)$, called the surrogate model, that provides the solution of the MSE at any spatial point $(x, y) \in \Omega$, frequency $\omega \in I_{\omega}$ and incoming wave angle $\theta \in I_{\theta}$, for fixed bathymetry and geometry of the harbor. Note that this function is defined in a 4dimensional coordinate system and, therefore, it must verify the MSE on the generalized domain $\Omega \times I_{\omega} \times I_{\theta}$. In weak form, this problem formalizes to

$$
A(\phi, v)=L(v) \text { for all } v(x, y, \omega, \theta) .
$$


The generalized bilinear and linear operators $A$ and $L$ are defined as

$$
\begin{aligned}
A(\phi, v) & =\int_{I_{\theta}} \int_{I_{\omega}} a(\phi, v ; \omega) d \omega d \theta \\
L(v) & =\int_{I_{\theta}} \int_{I_{\omega}} l(v ; \omega, \theta) d \omega d \theta
\end{aligned}
$$

where the implicit dependence on $\omega$ and $\theta$ is highlighted in $a(\cdot, \cdot)$ and $l(\cdot)$ that were defined in equations (7b) and (7c).

In order to solve the generalized problem (8) and obtain the surrogate model, the proper generalized decomposition solver (PGD) is applied. This method approximates the solution in the form of a separable expansion with $n$ terms,

$$
\phi(x, y, \omega, \theta) \approx \phi^{n}(x, y, \omega, \theta)=\sum_{m=1}^{n} F_{1}^{m}(x, y) F_{2}^{m}(\omega) F_{3}^{m}(\theta) .
$$

The PGD solver must provide all the unknown separable functions $F_{1}^{m}, F_{2}^{m}$ and $F_{3}^{m}$ for all the terms $m=1, \ldots, n$. The computation of these functions defines the offline phase of the MOR strategy, and the rapid evaluation of (9) for any spatial point in $\Omega$ and any spectral component in $I_{\omega} \times I_{\theta}$ denotes the online phase. The proposed MOR strategy consists, therefore, in using the PGD to compute the offline phase only once for a fixed harbor problem (i.e. geometry and bathymetry), and next performing extremely fast online evaluations to generate the required number of MSE solutions by the engineering applications, with minimal computer needs and computational time. In the following, the PGD rationale used to compute the offline phase and its associated computational cost is explained. For deeper details, the reader is addressed to [6] and the references therein.

The terms in the expansion (9) are evaluated using a greedy algorithm, in which each new term is found using the information contained in the previous $n-1$ terms, that are assumed to be known. The corresponding equation results from rewriting (9) as

$$
\phi^{n}(x, y, \omega, \theta)=\phi^{n-1}(x, y, \omega, \theta)+F_{1}(x, y) F_{2}(\omega) F_{3}(\theta),
$$

where the product $F_{1} F_{2} F_{3}$ denotes the unknown term of the expansion to be computed. Substituting $\phi$ by $\phi^{n}$ into (8a) and using expression (10) yields the problem

$$
A\left(F_{1} F_{2} F_{3}, v\right)=L^{n-1}(v) \text { for all } v(x, y, \omega, \theta),
$$

for the functions $F_{1}(x, y), F_{2}(\omega)$ and $F_{3}(\theta)$, where

$$
L^{n-1}(v)=L(v)-\sum_{m=1}^{n-1} A\left(F_{1}^{m} F_{2}^{m} F_{3}^{m}, v\right),
$$

accounts for the contribution of the previous $n-1$ terms. Thus, solving the 4-dimensional problem (11) provides the best new term (i.e. the best separable functions $F_{1}, F_{2}$ and $F_{3}$ ) 
to be incorporated into the expansion in order to improve the surrogate model. One of the main advantages of the PGD method is that it solves (11) efficiently by finding solutions on the so-called tangent space, that is

$$
v=v_{1} F_{2} F_{3}+F_{1} v_{2} F_{3}+F_{1} F_{2} v_{3}
$$

where $v_{1}(x, y), v_{2}(\omega)$ and $v_{3}(\theta)$ are test functions defined on separated dimensions. Using equation (12), the solution can be found using the following iterative process: at iteration $\gamma$, the function $F_{1}^{\gamma}$ is evaluated by solving the spatial equation

$$
A\left(F_{1}^{\gamma} F_{2}^{\gamma-1} F_{3}^{\gamma-1}, v_{1} F_{2}^{\gamma-1} F_{3}^{\gamma-1}\right)=L^{n-1}\left(v_{1} F_{2}^{\gamma-1} F_{3}^{\gamma-1}\right) \text { for all } v_{1}(x, y),
$$

where $F_{2}^{\gamma-1}$ and $F_{3}^{\gamma-1}$ denotes the known frequency and direction functions that come from the previous iteration. Once the solution $F_{1}^{\gamma}$ of this equation is found, it is used to update the value of $F_{2}^{\gamma-1}$ to $F_{2}^{\gamma}$ by solving the equation

$$
A\left(F_{1}^{\gamma} F_{2}^{\gamma} F_{3}^{\gamma-1}, F_{1}^{\gamma} v_{2} F_{3}^{\gamma-1}\right)=L^{n-1}\left(F_{1}^{\gamma} v_{2} F_{3}^{\gamma-1}\right) \text { for all } v_{2}(\omega),
$$

and finally, both values $F_{1}^{\gamma}$ and $F_{2}^{\gamma}$ are used to update the value of $F_{3}^{\gamma-1}$ to $F_{3}^{\gamma}$ by solving the equation

$$
A\left(F_{1}^{\gamma} F_{2}^{\gamma} F_{3}^{\gamma}, F_{1}^{\gamma} F_{2}^{\gamma} v_{3}\right)=L^{n-1}\left(F_{1}^{\gamma} F_{2}^{\gamma} v_{3}\right) \text { for all } v_{3}(\theta) .
$$

This process requires an initial guess for $F_{2}$ and $F_{3}$, which usually are selected randomly, and then equations (13a), (13b) and (13c) are solved repeatedly until the actual term of the surrogate model (i.e. the product $\left.F_{1} F_{2} F_{3}\right)$ converges. Note that equations $(13 \mathrm{~b})$ and $(13 \mathrm{c})$ are algebraic problems with negligible cost to be solved in the 1D parametric domains $I_{\omega}$ and $I_{\theta}$ respectively, whereas (13a) corresponds to a slight modification of the original MSE weak problem (7). Therefore, the offline computational cost associated to the PGD method is the product of: (i) the number of iterations, (ii) the final number of terms of the surrogate model (9), and (iii) the cost of one MSE simulation. It it worth noting here that any standard discretization method can be used to solve the equation (13a). However, the use of NEFEM, described in the previous section, is highly recommended because it can decrease drastically the latter cost and, consequently, the total cost of computing the surrogate model.

Finally an important remark must be done. The previous iterative scheme describes a standard PGD approach for clarity purposes. Nevertheless, for parameterized wave problems in frequency domain it is more convenient to use a Petrov-Galerkin scheme, namely PG PGD [12]. Similar computational costs are produced for one iteration if equation (13a) is evaluated with direct solvers, but the PG PGD usually generates less terms in the surrogate model [6]. Thus, without loss of generality, all the results presented in the following sections are obtained with the PG PGD method.

\subsubsection{Incremental offline construction of surrogate models with PGD}

For midrange wave periods (10 to $16 \mathrm{~s}$ ) and medium-sized harbors, the standard offline construction of surrogate models usually needs some hundreds of MSE solutions, which 


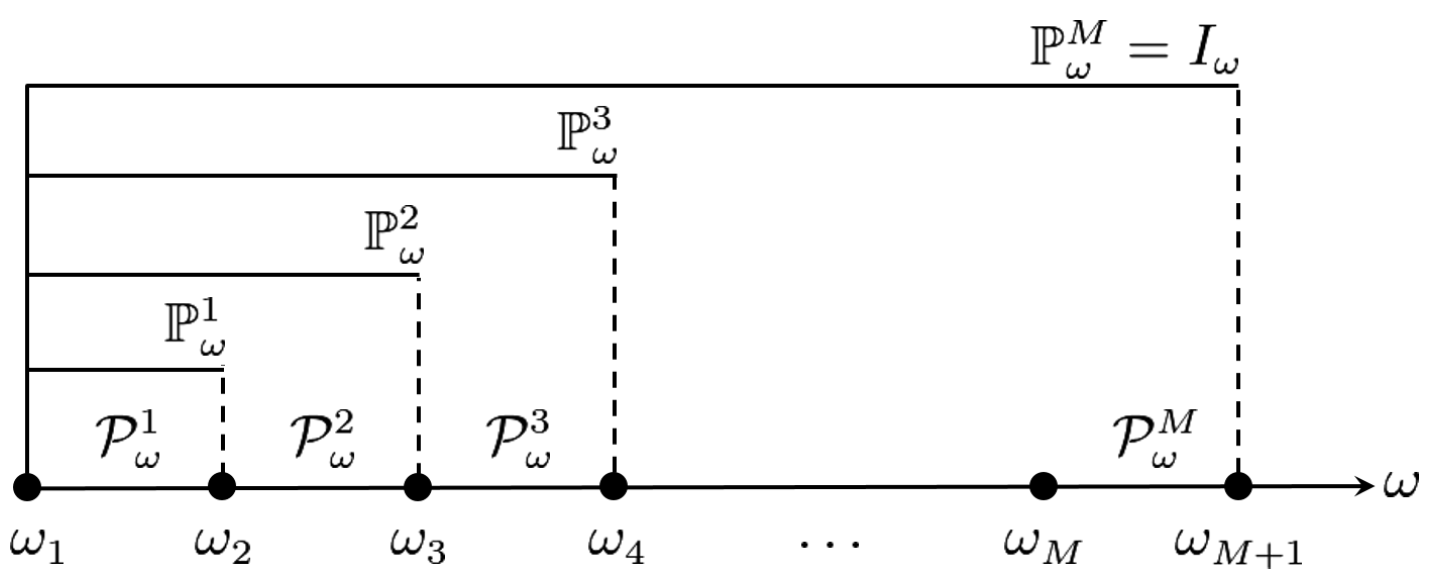

Figure 3: Incremental partitions of frequency domain $I_{\omega}=\left[\omega_{1}, \omega_{M+1}\right]$.

may increase to several thousands if large harbors are of concern, see some examples in [6]. However, some harbor applications require lower wave periods. Unfortunately, in these cases (e.g. $6 \mathrm{~s}$ of wave period) standard PGD algorithms lose convergence and may fail in providing solutions, limiting the applicability of the MOR strategy. As a first attempt to overcome this issue, a new practical procedure to construct surrogate models with PGD, based on incremental computations along the frequency dimension, is presented next. It follows a similar strategy to those encountered in the literature that accelerate convergence to global minima for wave propagation problems, for instance when solving inverse modeling with multiscale methods along the frequency domain [39].

Some notation and definitions are required first. Let $\omega_{a}$ and $\omega_{b}$ be the lower and the higher frequencies of interest, respectively, for the harbor agitation problem. Then, let us truncate the 1D frequency domain $I_{\omega}=\left[\omega_{a}, \omega_{b}\right]$ into $M$ partitions or subdomains $\mathcal{P}_{\omega}^{j}=\left[\omega_{j}, \omega_{j+1}\right]$ for $j=1, \ldots, M$. Note that $\omega_{1}=\omega_{a}, \omega_{M+1}=\omega_{b}$ and $\omega_{j}<\omega_{j+1}$. Let us also define, for each partition $\mathcal{P}_{\omega}^{j}$, the frequency band that covers all the frequencies from $\omega_{a}$ to the end of the partition, $\omega_{j+1}$. Formally, this is denoted by $\mathbb{P}_{\omega}^{j}=\bigcup_{r=1}^{j} \mathcal{P}_{\omega}^{r}$, where $\mathbb{P}_{\omega}^{j} \subset \mathbb{P}_{\omega}^{j+1}$. For clarity purposes, all these truncations of the original frequency domain $I_{\omega}$ are illustrated in figure 3 .

Using these partitions, the surrogate model $\phi$ is proposed to be defined here as

$$
\phi=\phi_{1}+\phi_{2}+\ldots+\phi_{M},
$$

where $\phi_{j}(x, y, \omega, \theta), j=1, \ldots, M$ is a surrogate model for $(x, y) \in \Omega, \omega \in \mathbb{P}_{\omega}^{j}$ and $\theta \in I_{\theta}$. Each of these surrogate models provides the MSE solution for a frequency band that increases incrementally from $\phi_{j}$ to $\phi_{j+1}$. Thus, this strategy is coined as an incremental offline construction where $\phi_{1}$ propagates only the smallest frequencies while $\phi_{M}$ includes all the frequencies in $I_{\omega}$. A natural and efficient strategy to construct $\phi$ using (14) follows a greedy procedure, where each surrogate model $\phi_{j}$ is constructed from the information contained in all the previous surrogate models $\phi_{1}, \ldots, \phi_{j-1}$. Essentially, this is the same 
greedy strategy used by the PGD method to compute each term in equation (10). Formally, this implies solving the following $M$ problems

$$
A\left(\phi_{j}, v\right)=L(v)-\sum_{r=1}^{j-1} A\left(\phi_{r}, v\right) \quad \text { for } j=1, \ldots, M,
$$

for all functions $v(x, y, \omega, \theta)$. In practice, solving the $j$-th equation in (15) is straightforward: it means applying the PGD method explained in the previous section to construct $\phi_{j}$, but initializing the greedy process in (10) at $n=1$ with the terms corresponding to all the previous surrogate models already computed, i.e. $\phi_{1}, \ldots, \phi_{j-1}$. Convergence for higher frequencies can be improved with the incremental construction because solutions to previous frequency bands are provided in the right hand side of (15). Moreover, in contrast to similar PGD approaches in the literature, like the recent local PGD algorithm [40], this strategy generates only one expansion in the form of equation (9) that can be efficiently compressed a posteriori, see [6], if the produced number of terms is very large. Results of section 4 show that this strategy provides solutions in cases when the standard application of PGD fails.

\section{Application to wave agitation in harbors}

This section applies the proposed numerical strategy to several harbor application examples, in which the engineering quantity of interest generally is the amplification factor at any point of the computational domain, namely

$$
H(x, y)=|\phi(x, y)| / A_{0},
$$

where $A_{0}$ is the amplitude of the offshore incoming wave. This is set to $A_{0}=1$ in all the examples due to the linearity of the MSE model.

Convergence criteria for the PGD solver are specified following standard proxies for termination and a tolerance $\epsilon$. Firstly, for the iterative process of equations (13) at iteration $\gamma$, this implies

$$
\frac{\left\|F_{1}^{\gamma} F_{2}^{\gamma} F_{3}^{\gamma}-F_{1}^{\gamma-1} F_{2}^{\gamma-1} F_{3}^{\gamma-1}\right\|_{\mathcal{L}^{2}}^{2}}{\left\|F_{1}^{\gamma} F_{2}^{\gamma} F_{3}^{\gamma}\right\|_{\mathcal{L}^{2}}^{2}}<\epsilon^{2},
$$

where the $\mathcal{L}^{2}$ norm applies on the 4-dimensional domain $\Omega \times I_{\omega} \times I_{\theta}$ in case of the standard PGD constructor, or $\Omega \times \mathbb{P}_{\omega}^{j} \times I_{\theta}, j=1, \ldots, M$, in case of the incremental PGD described in section 3.2.1. Secondly, once convergence in (16) is achieved or a maximum number of iterations is reached (usually not higher than three [6]), convergence of the surrogate model (9) as the number of terms $n$ increases can be checked by

$$
\frac{\left\|\phi^{\text {ref }}-\phi^{n}\right\|_{\mathcal{L}^{2}}^{2}}{\left\|\phi^{n}\right\|_{\mathcal{L}^{2}}^{2}}<\epsilon^{2}
$$


where $\phi^{\text {ref }}(x, y, \omega, \theta)$ denotes a reference solution that can be computed using brute force approaches, for instance, using either FEM or NEFEM at each sample of frequency and incoming wave direction. When neither this reference is available nor the error (17a) is computable, the usual way to measure the quality of the PGD approximation is

$$
\frac{\left\|F_{1} F_{2} F_{3}\right\|_{\mathcal{L}^{2}}^{2}}{\left\|\phi^{n-1}\right\|_{\mathcal{L}^{2}}^{2}}<\epsilon^{2},
$$

that indicates the contribution of the last term on the PGD expansion. Equation (17b) usually provides efficient indicators that behave similarly to the error computation (17a) for low tolerances. However, see [41] in need of a more precise (and expensive) error estimator based on the computation of an adjoint solution.

\subsection{Long wave propagation and resonance in Marina di Carrara harbor}

The first application computes the long wave propagation in Marina di Carrara harbor, located in the Northwest of Italy. This case corresponds to the harbor resonance study by [15] with a constant interior bathymetry of $10.5 \mathrm{~m}$ and totally reflecting boundaries, i.e. $\alpha=0$ in equation (5). They performed a large number of simulations for a range of periods between $10000 \mathrm{~s}$ and $33 \mathrm{~s}$ in order to identify the resonance modes induced by the harbor geometry. In this situation the choice of a proper strategy providing efficient solutions can be crucial. For practical purposes, the worst case scenario is selected first to study the efficiency of the computational mesh. A particular incoming wave with $33 \mathrm{~s}$ of period and an incident direction of $\theta=225^{\circ}$ is used (recall that orientation of the angle $\theta$ is stated in figure 1). The problem statement and the reference solution of the amplification factor for this case are depicted in figure 4. This reference is obtained using a 4th order triangular mesh with a wave resolution of $226 \mathrm{npw}$ (i.e. nodes per wavelength). Observe that the geometry of this harbor contains small geometric details of size $h_{b}=15 \mathrm{~m}$.

In order to provide an efficient discretization minimizing the number of DOF for this case, one possible mesh choice and its simulation results are depicted in the top of figure 5 . It corresponds to large 4th order elements of size $h=150 \mathrm{~m}$ providing roughly uniform wave resolution of $8 \mathrm{npw}$ and $671 \mathrm{DOF}$. This example demonstrates how an inadequate description of the boundary clearly deteriorates the simulation in cases where $h_{b} \ll h$, producing wrong results even if sufficient wave resolution is used. Recall that first order meshes are not recommended because of their low cost/benefit ratio in wave problems compared to higher order approximations [36]. The standard use of high order meshing algorithms to solve this issue is limiting the size of boundary elements to $h=h_{b}$, which usually is a strong condition producing severe refinements if $h_{b} \ll h$. An example is depicted in figure 5 with a 4 th order adapted mesh that generates accurate results, but substantially increasing the DOF compared to a uniform mesh size $h=150 \mathrm{~m}$. In order to retain sufficient accuracy on the uniform mesh, the last simulation depicted in figure 5 shows 

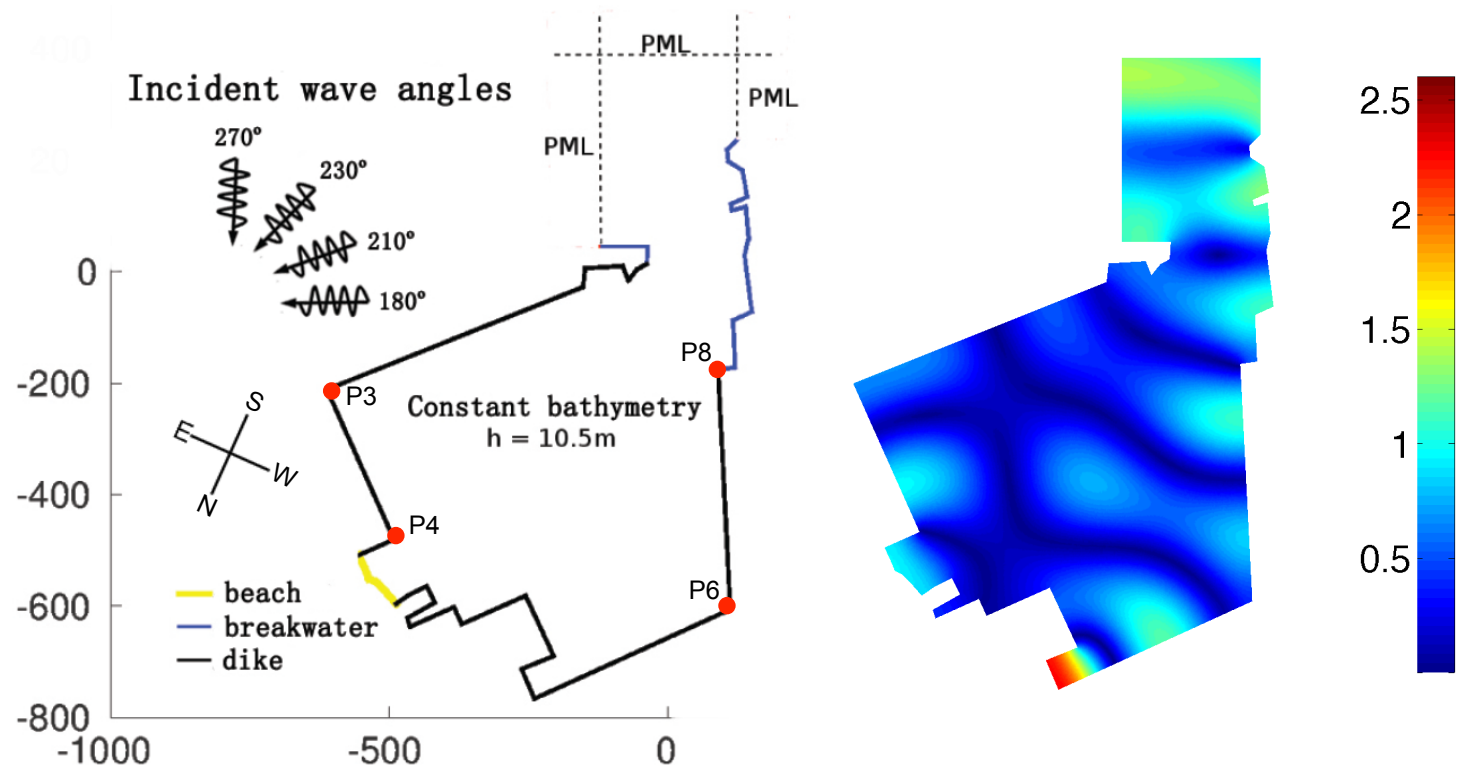

Figure 4: Carrara harbor: definition of problem statement (left) and reference solution of the amplification factor for an incident wave with $38 \mathrm{~s}$ of period and $225^{\circ}$ of incoming direction (right).

the result using NEFEM. Here, the large elements are able to capture the exact harbor boundaries regardless of their complexity and with no need of refinements or geometry simplifications. This exact description of the geometry for boundary elements overcomes the previous limitations and provides valid results using 5.5 times less DOF than the adapted mesh. It is worth noting that, in consequence, NEFEM improvement in efficiency can be relevant in processes requiring multitude of these simulations, for instance the resonance study performed in [15] which is replicated next using the proposed model order reduction strategy.

The PGD solver has been used to construct offline a surrogate model for this example. Since the resonance study requires numerous repetitive simulations on the same harbor geometry, this surrogate model is used online to generate these simulations with negligible computational cost for any spectral component, that is, any frequency $\omega$ and incoming wave angle $\theta$ of interest. According to [15] frequencies vary in $I_{\omega}=[0.00063,0.19] \mathrm{rad} / \mathrm{s}$, which is equivalent to wave periods from $10000 \mathrm{~s}$ to $33 \mathrm{~s}$. The range in variable $\theta$ covers all the incoming wave directions from southwest to southeast, i.e. $\theta \in I_{\theta}=\left[180^{\circ}, 270^{\circ}\right]$, see figure 4. In order to solve the 1D parametric problems (13b) and (13c) during the offline PGD construction, linear FEM is used with 2000 and 40 nodes to discretize $I_{\omega}$ and $I_{\theta}$ respectively (both grids are uniformly distributed). For the 2D problem (13a) NEFEM is used on the uniform 4th order mesh depicted in figure 5 to increase the efficiency as demonstrated before.

Figure 6 depicts the PGD errors and number of terms produced in the offline con- 

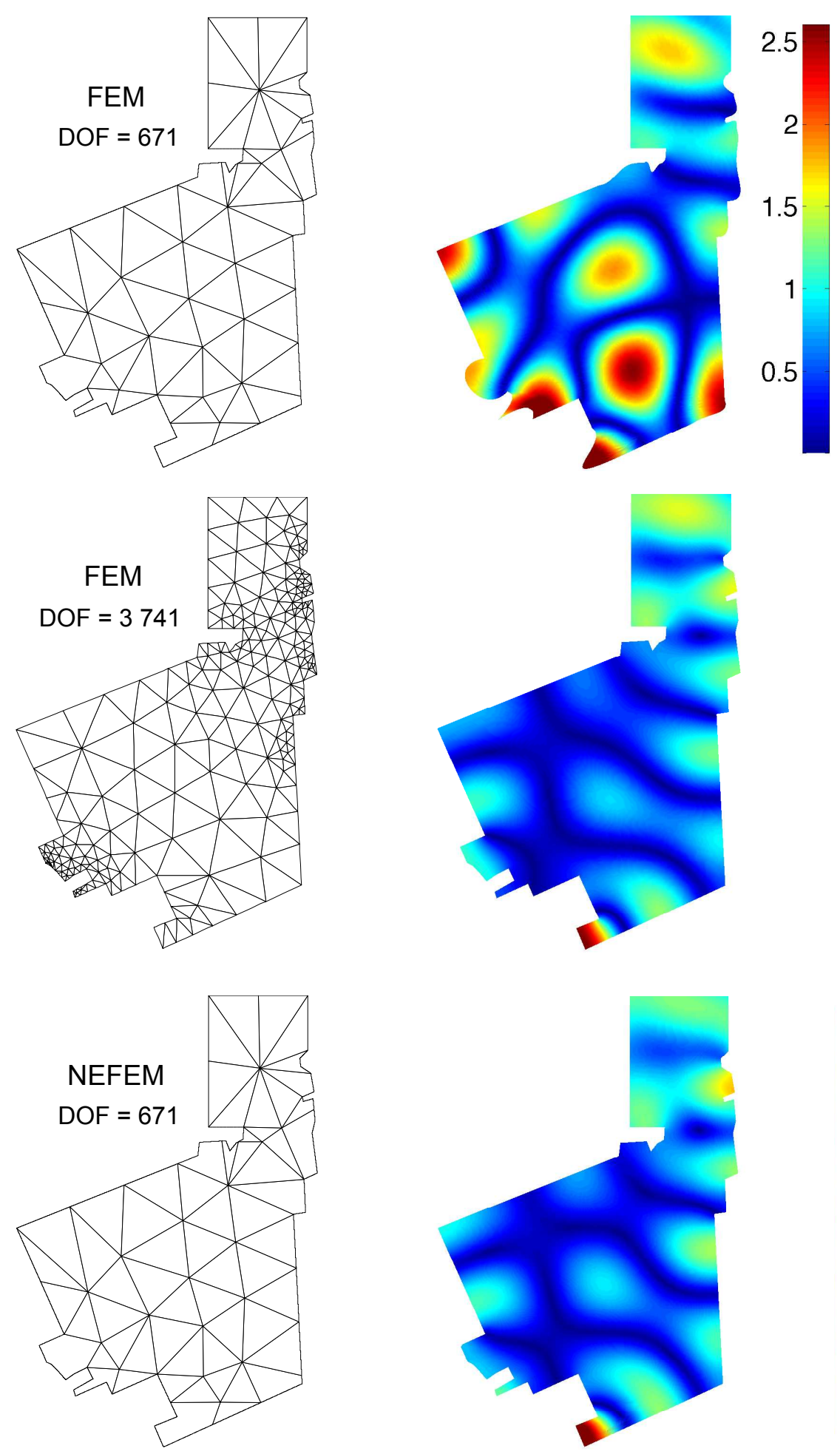

Figure 5: Carrara harbor: 4th order mesh and respective amplification factor for an incident wave with $38 \mathrm{~s}$ of period and $225^{\circ}$ of incoming direction. Cases shown are FEM with uniform mesh (top row), FEM with adapted mesh (mid row) and NEFEM with uniform mesh (bottom row). 

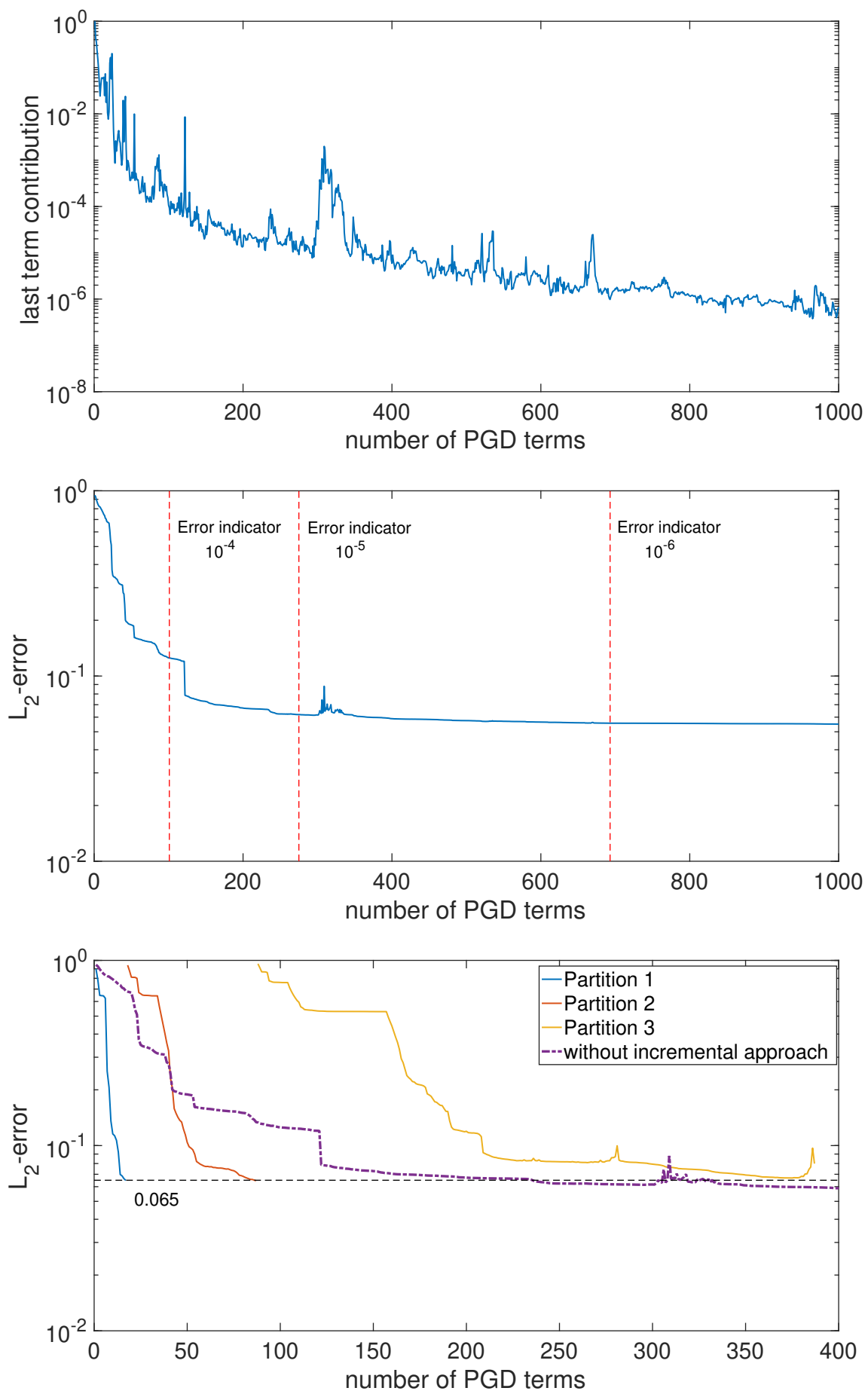

Figure 6: Long waves in Carrara harbor: PGD error indicator (top), $\mathcal{L}^{2}$ error (mid) and $\mathcal{L}^{2}$ error for incremental PGD (bottom). 

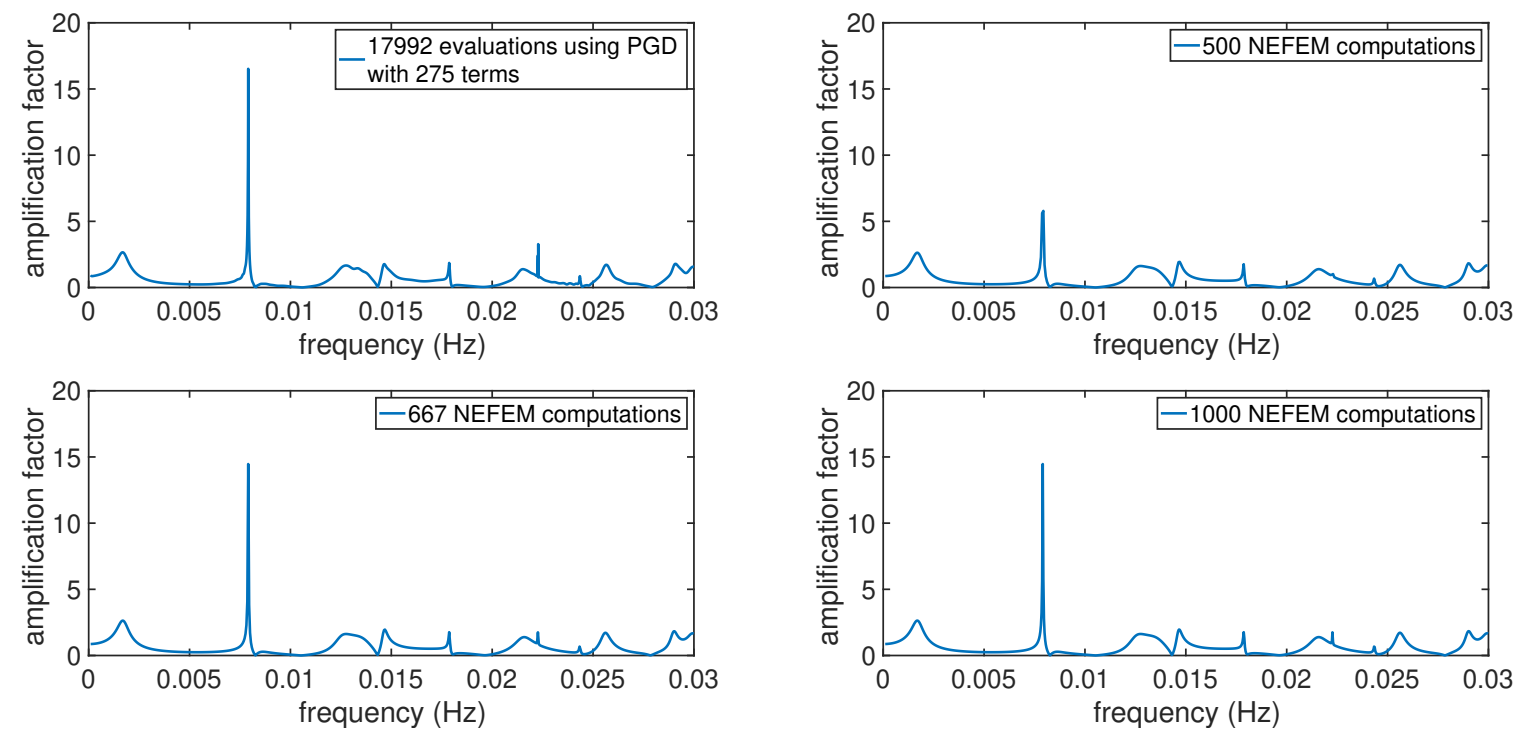

Figure 7: Carrara harbor: frequency spectrum at point P3, along the entire wave period range with fixed incoming wave direction $\theta=185^{\circ}$. The amplification factor is obtained with (i) the proposed MOR strategy (top left, by the online surrogate model evaluation with 275 terms), and (ii) the standard brute force approach, using here the NEFEM method at different number of spectral components: 500 (top right), 667 (bottom left) and 1000 (bottom right).

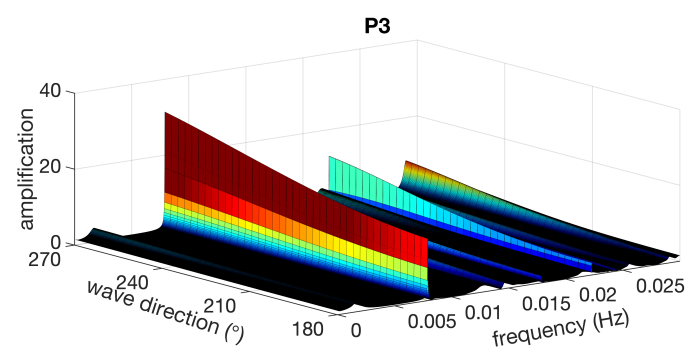

P6

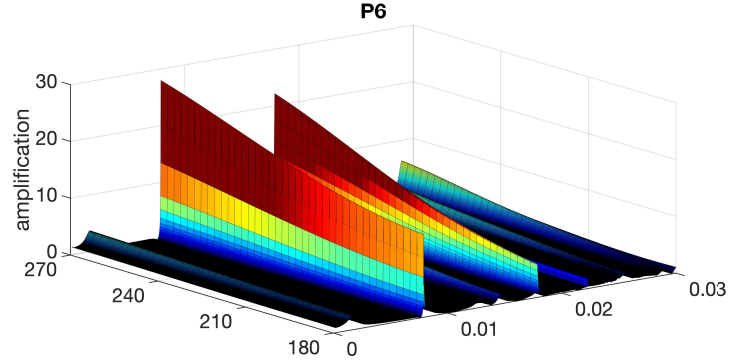

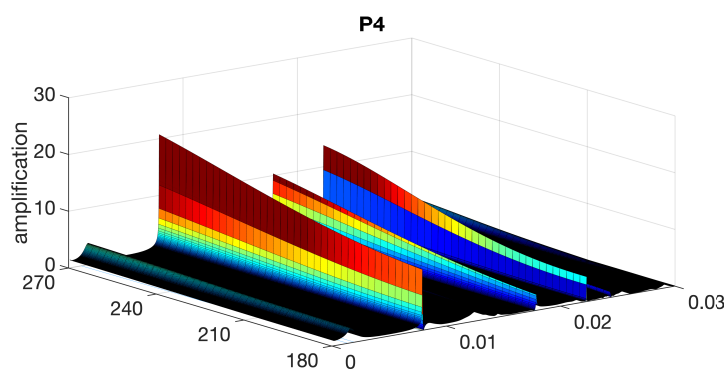

P8

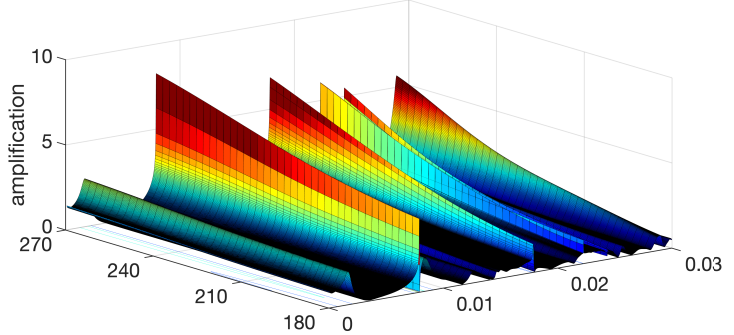

Figure 8: Carrara harbor: frequency-direction spectrum at points P3 to P8 obtained with the proposed MOR strategy. 
struction of the expansion (9) for this harbor. Convergence is obtained for both the error computation (17a) and the indicator (17b), revealing a saturated level of accuracy that is representative of MOR techniques. Results show that 275 terms in the expansion are enough to achieve a $6 \%$ of error in the solution with an indicator value of $10^{-5}$. The error obtained with the incremental PGD construction using three subdomains is also depicted in figure 6 and compared to the standard constructor. However, as expected in this low frequency problem, the incremental strategy is not relevant because the standard application imposes less terms to provide a surrogate model with the same level of accuracy.

The harbor resonance study is depicted in figures 7 to 9. The amplification factor is computed at spatial points P3, P4, P6 and P8 used in [15] and shown in figure 4. Recall that the harbor spectra are obtained with no relevant computational cost (i.e. with no need of solving any new simulation) by using the online evaluation of the already constructed surrogate model. Despite the resonance frequencies are generally not known a priori, the real-time execution of the online MOR phase allows high refinements of the spectra that capture the full resonance response. This is not the case in conventional approaches based on brute force computations because a new simulation must be computed for each new parameter, and thus some resonance frequencies may be ignored. This issue is illustrated in figure 7 for the spatial point P3. For fixed southwest direction of incident waves (see figure 4), the PGD expansion with 275 terms is rapidly evaluated at 17992 different frequencies. This is compared with the brute force approach using NEFEM solutions for three different frequency discretizations: 500, 667 and 1000 number of frequencies uniformly distributed in $I_{\omega}$. It can be observed that the resonance peak at $0.022 \mathrm{~Hz}$ is not obtained evaluating only 500 frequencies, and it is slightly captured with 667 . In fact, the latter case imposes a computational cost rather below to that required in the offline computation of the 275 PGD terms, but providing results for 26 times less number of frequencies. Note that this difference becomes drastic when adding different incoming wave directions to complete the resonance analysis, as it is illustrated in figure 8. Here, wave amplifications are depicted at the four spatial points for all the combinations in the parametric domain $I_{\omega} \times I_{\theta}$. Unlike standard brute force approaches that usually evaluate a few incoming waves, the resonance frequencies are clearly observed in figure 8 for the full range of incident directions, and with no relevant extra times due to the fast online calculation. These results highlight the potential of the proposed MOR strategy for this variety of applications in harbor agitation problems.

For comparison purposes, the frequency spectra computed from amplification factors are also depicted in figure 9. The accuracy of the online evaluation using the MOR strategy is compared to the reference solution using the standard brute force approach (i.e. solving the MSE at each spectral component with, in this case, NEFEM). In both cases 2000 simulations have been used to generate the spectra. Note that the overall agreement is perfectly captured for the entire frequency range. 

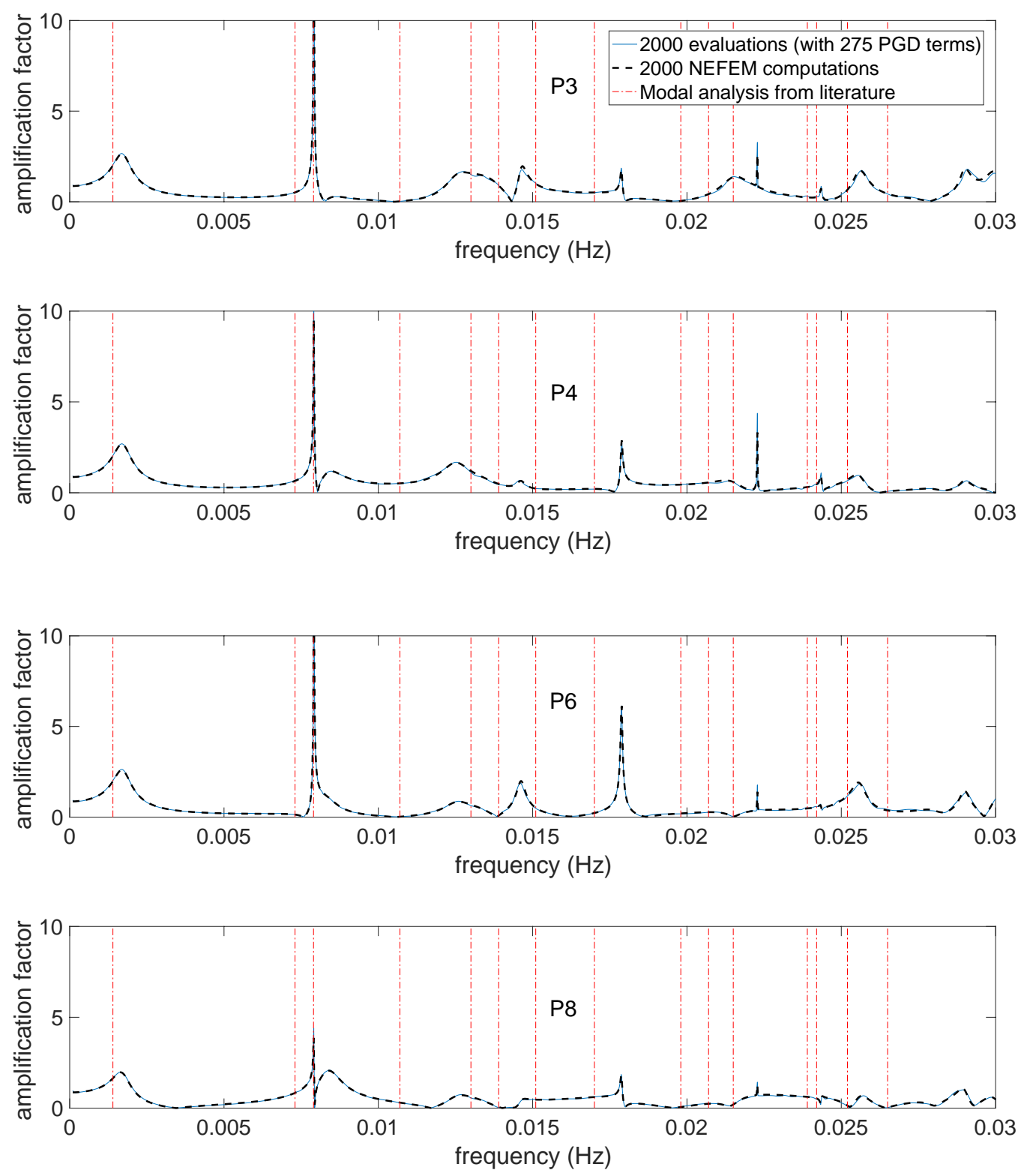

Figure 9: Carrara harbor: frequency spectrum from amplification factor. Comparison at points $\mathrm{P} 3, \mathrm{P} 4, \mathrm{P} 6$ and $\mathrm{P} 8$ for fixed incoming wave direction $\theta=185^{\circ}$. The solution of the MOR strategy with PGD is compared to the brute force approach where each spectral computation is performed with NEFEM. The modal analysis proposed in [15], that estimates the main harbor modes, is also shown for information purposes. 

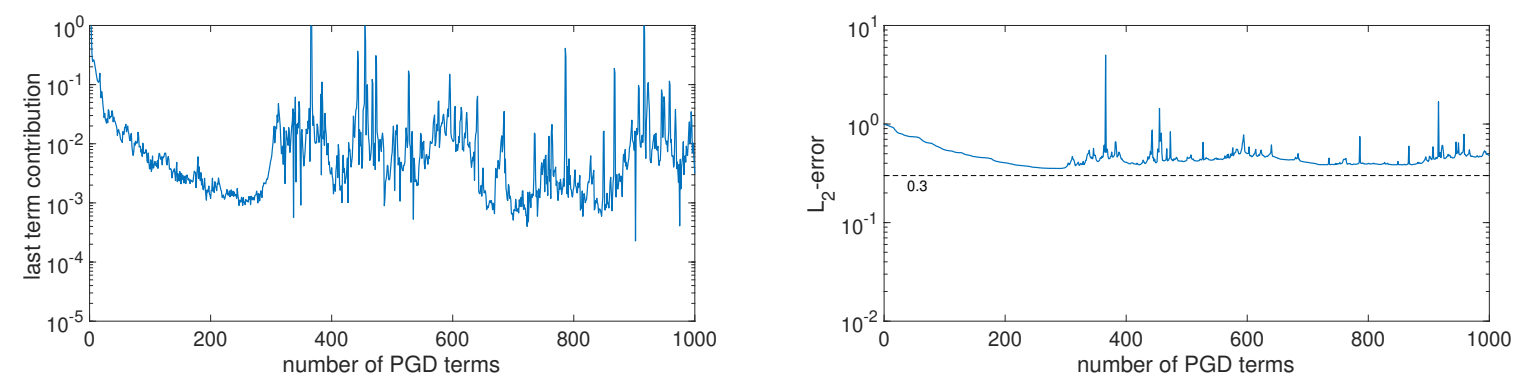

Figure 10: Short waves in Carrara harbor: error indicator (left) and $\mathcal{L}^{2}$ error (right) for the standard PGD constructor.

\subsection{Short wave propagation in Marina di Carrara harbor}

The second application deals with the short wave propagation in Marina di Carrara harbor. The same geometry depicted in figure 4 is used with absorption coefficients $\alpha=\{0.43,0.72,0.05\}$ for breakwater, beach and dike boundaries respectively [6]. In this case solutions of interest are seek for any wave period in the range $4 \mathrm{~s}$ to $14 \mathrm{~s}$ and any incoming wave from southwest directions, that is $\omega \in[0.45,1.57] \mathrm{rad} / \mathrm{s}$ and $\theta \in\left[210^{\circ}, 230^{\circ}\right]$. The spatial discretization corresponds to a 4th order mesh with 176554 DOF and a minimum wave resolution of $10 \mathrm{npw}$. It is worth noting that the generated mesh size is of the same order than the minimum boundary size, and consequently FEM and NEFEM approaches produce similar results in this case.

Due to the higher design frequencies of this problem, the standard PGD constructor fails in providing an accurate surrogate model as shown in figure 10, where the PGD expansion does not converge for neither of the expressions in (17). Therefore, in contrast to the long wave propagations solved in the previous example, this problem provides a test case for the incremental PGD constructor proposed in section 3.2.1.

Several partition strategies are tested, in particular, the frequency domain is truncated into $M=\{4,5, \ldots, 10\}$ different number of partitions uniformly distributed in $I_{\omega}$. The resulted errors (17a) are depicted in figure 11. It can be observed how the incremental strategy is able to reduce the $40 \%$ of error produced by the standard PGD constructor (recall figure 10) to $9 \%$ by using only $M=4$ partitions. In fact, the incremental constructor recovers the PGD convergence in all the frequency partitions, as depicted in figure 11 for the case $M=10$. Note that this is the optimal case able to reach a goal error of $5 \%$ with 3259 terms in the PGD expansion. The accuracy of the online generated wave amplification factor is exemplified in figure 12 for the particular case of $\omega=1.57 \mathrm{rad} / \mathrm{s}$ and $\theta=201^{\circ}$ using the FEM solution as the reference. Major differences are produced in the wave height on those areas of higher reflections, while the wave phase remains under general good agreement. This coincides with previous works on PGD for scattering wave problems [6].

Finally, to alleviate the online evaluation and storage of the surrogate model, the final 

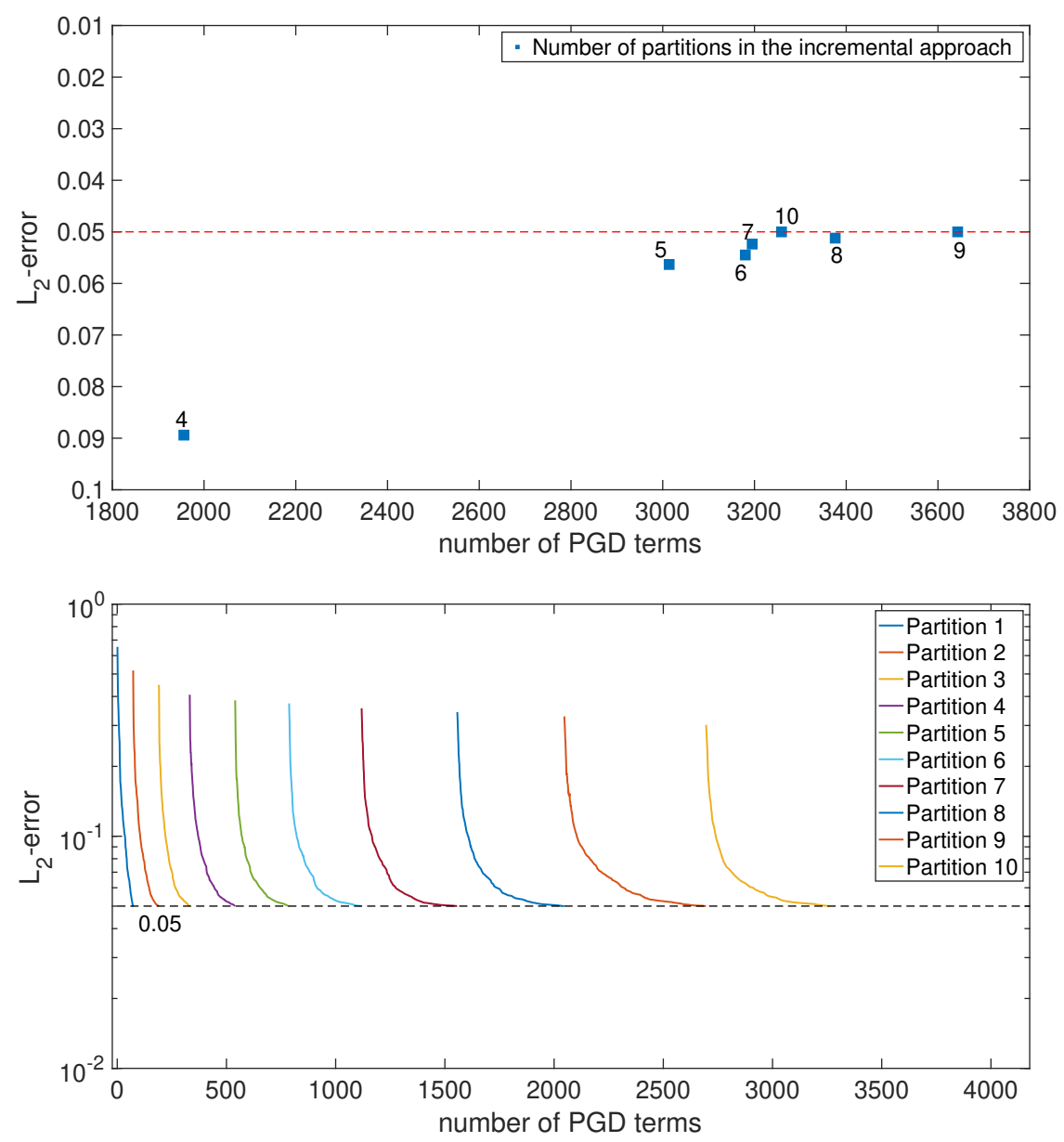

Figure 11: Short waves in Carrara harbor: $\mathcal{L}^{2}$ error and number of PGD terms for the incremental strategy in the frequency dimension. It is depicted as the final error reached for different number of frequency partitions (top), and as the error convergence for the particular case of 10 partitions (bottom).

number of terms can be reduced with marginal costs using the PGD-projection technique presented in [6]. For instance, results for the case $M=10$ indicate a compression rate of nearly 14 that provides 240 PGD terms (not shown in this paper).

\subsection{Barcelona harbor}

The MOR strategy for large spatial domains, variable bathymetry and higher frequencies is applied in this last example. The Barcelona harbor model depicted in figure 13 is used for design wave periods ranging from $6 \mathrm{~s}$ to $12 \mathrm{~s}$, and south incoming wave directions. Absorption coefficients in this case are $\alpha=\{0.43,0.05\}$ for breakwater and dike boundaries respectively. Some details of the spatial mesh on three different areas of the harbor are also 

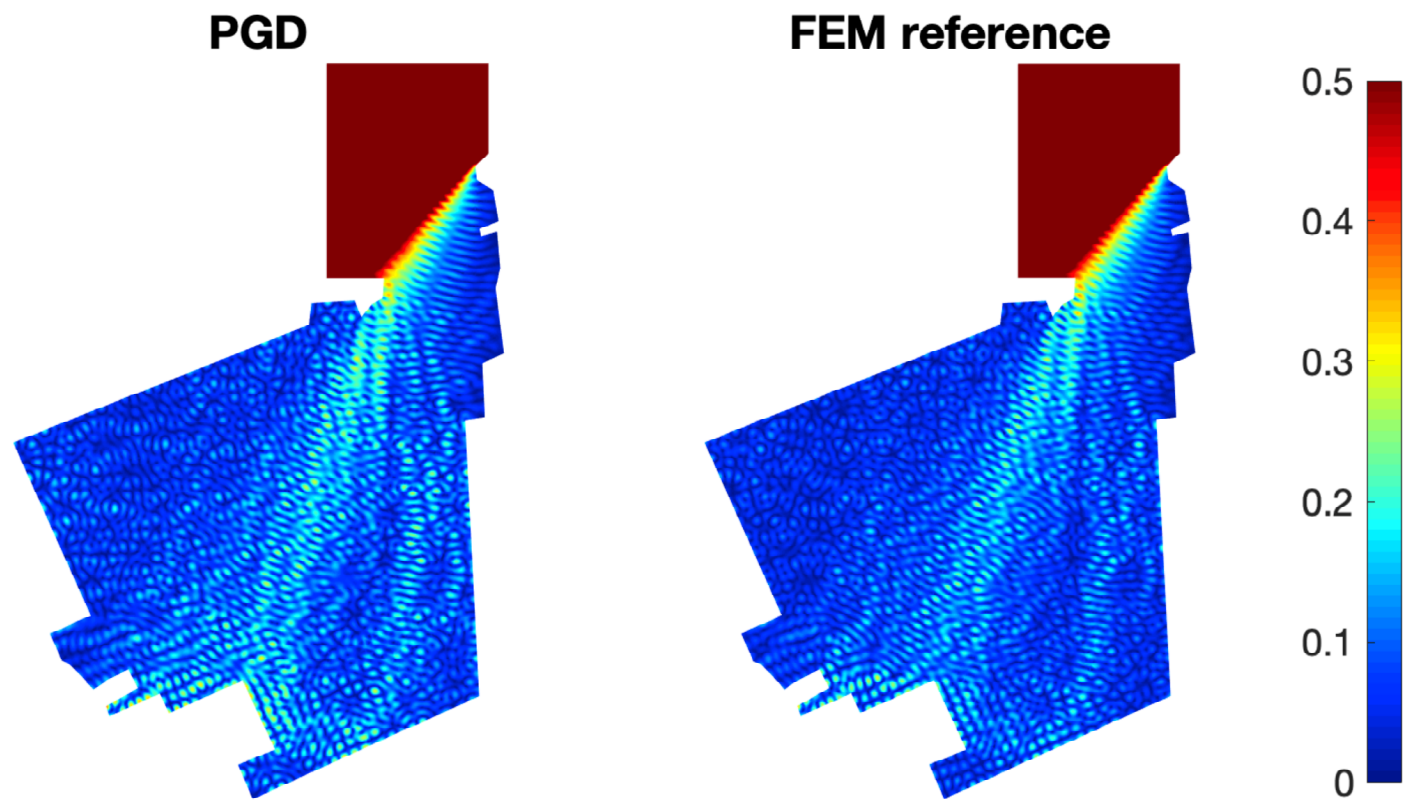

Figure 12: Short waves in Carrara harbor: wave amplification comparison between high order FEM reference (left) and PGD solution (right) for frequency $\omega=1.57 \mathrm{rad} / \mathrm{s}$ and incoming wave direction $\theta=229^{\circ}$.

shown in the same figure. They correspond to a 4th order discretization with a minimum wave resolution of $8 \mathrm{npw}$ and 1.5 millions of DOF. In the offline MOR phase with PGD, both frequency and incoming wave direction domains $\left(I_{\omega}\right.$ and $\left.I_{\theta}\right)$ are discretized with linear elements using 300 and 40 nodes respectively.

In order to retain sufficient convergence of the PGD method in this large-scale example, the frequency domain $I_{\omega}$ is partitioned into 60 equally-spaced subdomains when performing the incremental PGD construction. Since the high dimensional size of the problem precludes the computation of a reference solution, only the error indicator (17b) is used for convergence criteria. Results are depicted in figure 14 for the particular case of partition 8 (lower frequencies), 24, 50 and 60 (higher frequencies). Although, as expected, the convergence rate degrades as long as the frequency increases, the error indicator can achieve a solid tolerance of $10^{-4}$ which provides sufficient precision for the online evaluation of the surrogate model. The total number of terms computed in the PGD expansion for this example are 12 000, which can be compressed a posteriori to a final number of 1613 terms. In figure 15 some of these online results are compared to a FEM reference for several input parameters and areas of the harbor. Note that wave amplifications are well captured in general, especially the wave phases (less than $10 \%$ of spatially-averaged relative error), and the larger errors are produced for the shorter waves and the most reflective area (see region $\mathrm{Z} 2$ in figure 13). 

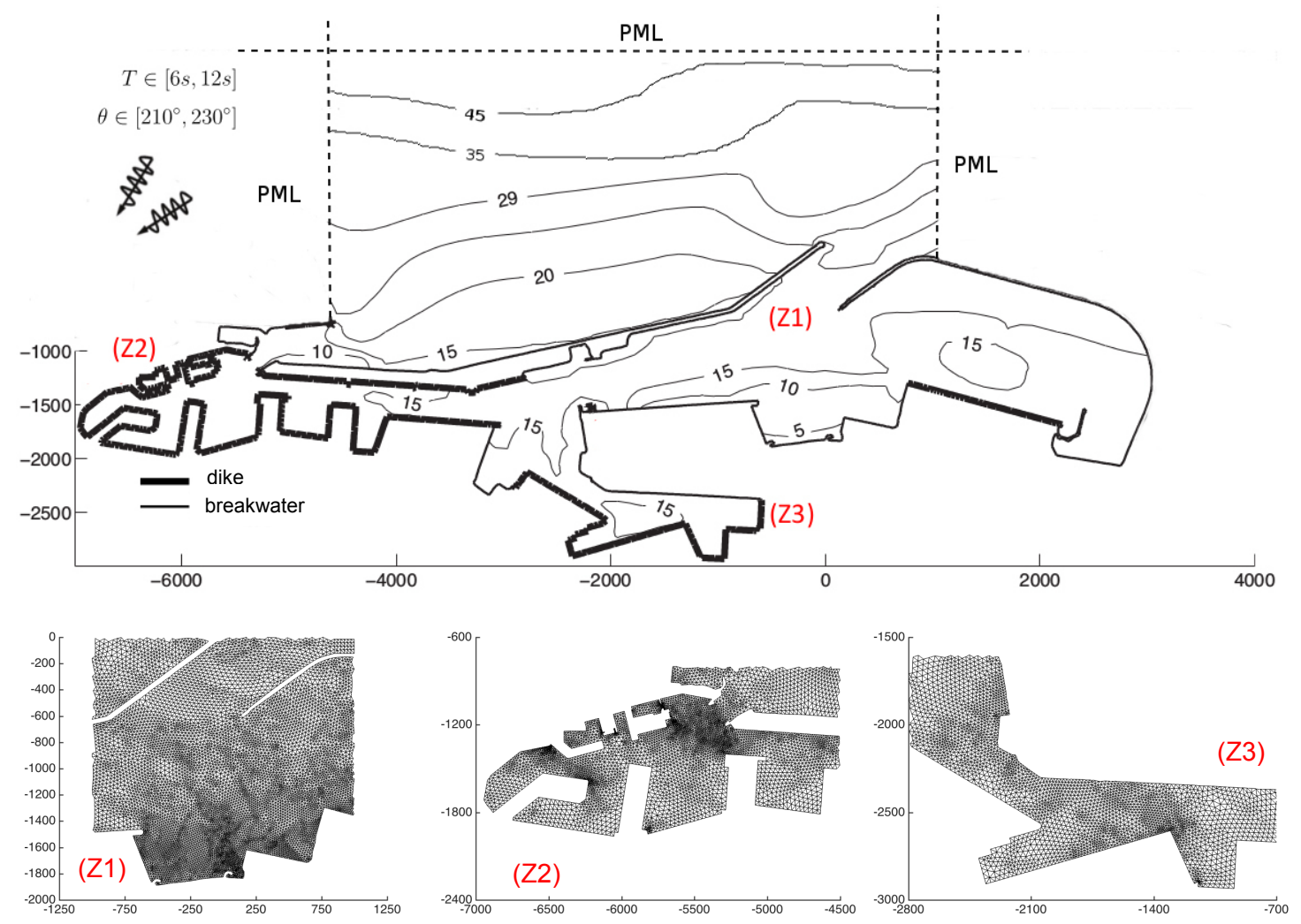

Figure 13: Barcelona harbor: definition of geometry, bathymetry, and range of design wave periods and incoming wave directions (top). Some mesh details are depicted in three different harbor areas (bottom).

\section{Concluding remarks}

An online-offline MOR strategy, combined with NEFEM computations for the offline phase, is proposed to solve elliptic harbor agitation models driven by many parametric scenarios, typically, numerous evaluations of the frequency-direction spectra. On the one hand, NEFEM avoids the necessity of local refinements in spatial discretizations that are excessively dominated by the geometric representation of the boundary. For instance, in long wave propagation cases NEFEM provides a significant reduction in DOF while retaining sufficient level of accuracy.

On the other hand, the online evaluation of the surrogate model produced by the PGD method enables an extremely fast calculation of solutions for any spectral component, i.e. frequency and incoming wave direction. The efficiency in terms of computational cost and accuracy of this strategy is illustrated with a resonance study in Marina di Carrara harbor, Italy. The resonant response of the harbor is very well captured with no limits on the number of evaluated parameters, overcoming the standard brute force approach used 


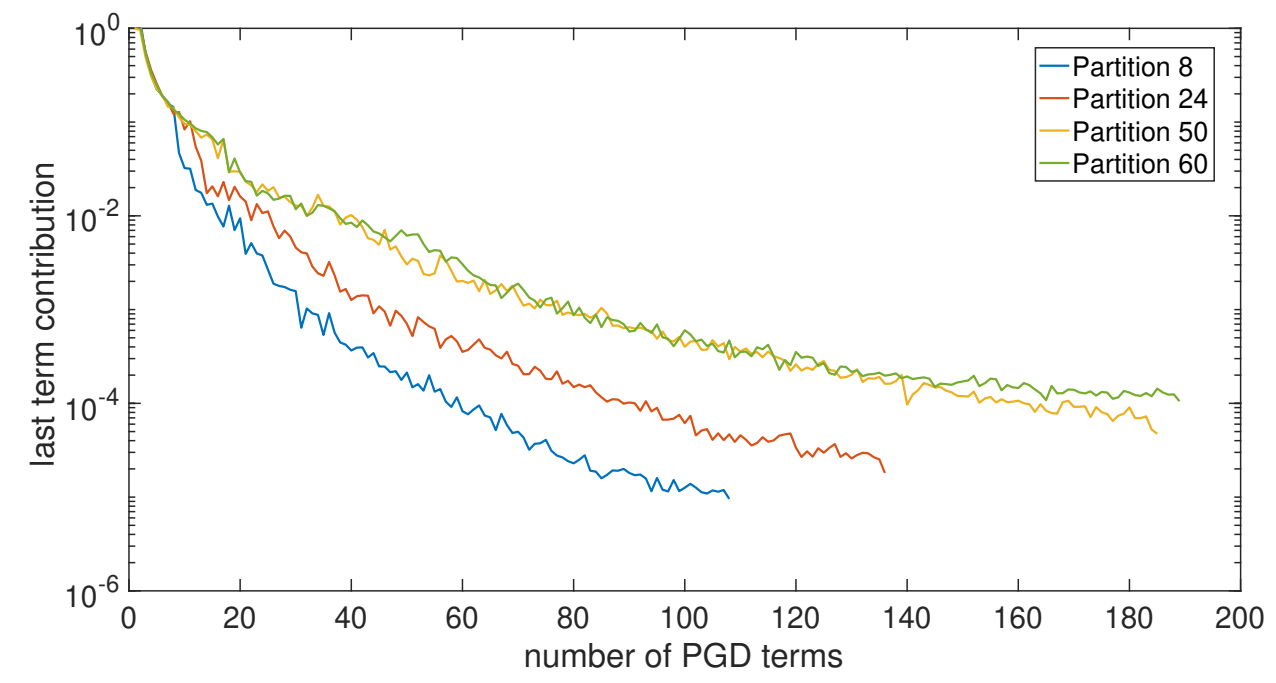

Figure 14: Barcelona harbor: error indicator for incremental PGD at different partitions of the frequency domain.

in practice.

A novel incremental approach to compute the surrogate model within the PGD rationale is also presented. It is oriented to retain the convergence of the PGD expansion for cases in which higher frequency ranges are of interest. Short wave propagation results in Marina di Carrara harbor demonstrate that the new incremental approach provides convergence in cases where the standard PGD constructor fails. As a final application, the proposed strategy is used to solve a high frequency range problem in the Barcelona harbor. The produced surrogate model exemplifies the potential of the MOR strategy to generate fast solutions for large scale harbor agitation problems.

\section{Acknowledgments}

David Modesto thanks the funding of this project through the GEOTHERMICA ERANET co-fund (project no. 731117) and the Spanish Ministry project PCI2018-093186. Antonio Huerta and Sergio Zlotnik thank the funding from the Generalitat de Catalunya SGR 2017-2019 and the project DPI2017-85139-C2-2-R funded by the Spanish Ministry. Boyi Ye is funded by the European Commission EACEA Agency, Framework Partnership Agreement 2013-0043 Erasmus Mundus Action 1b, as a part of the EM Joint Doctorate Simulation in Engineering and Entrepreneurship Development (SEED). 

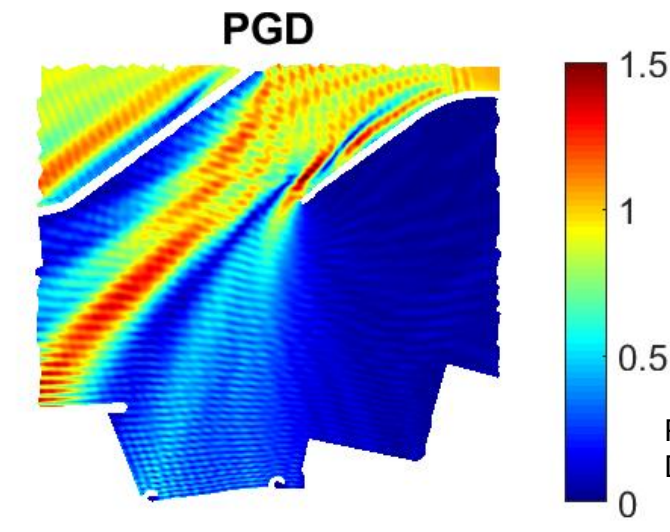

PGD
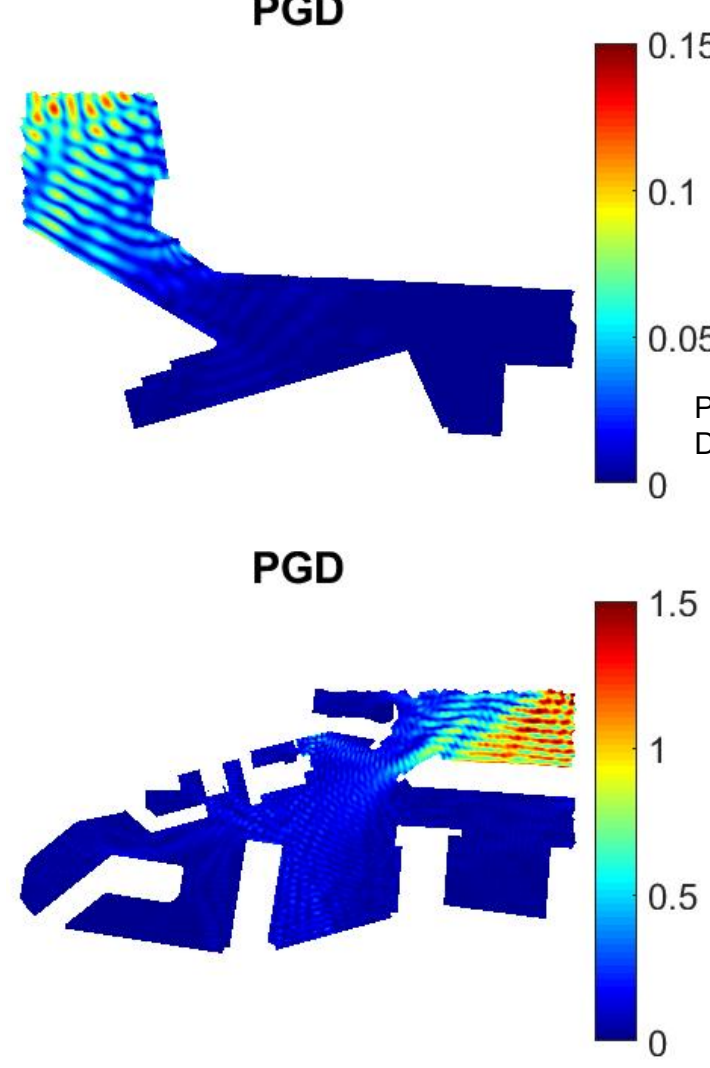

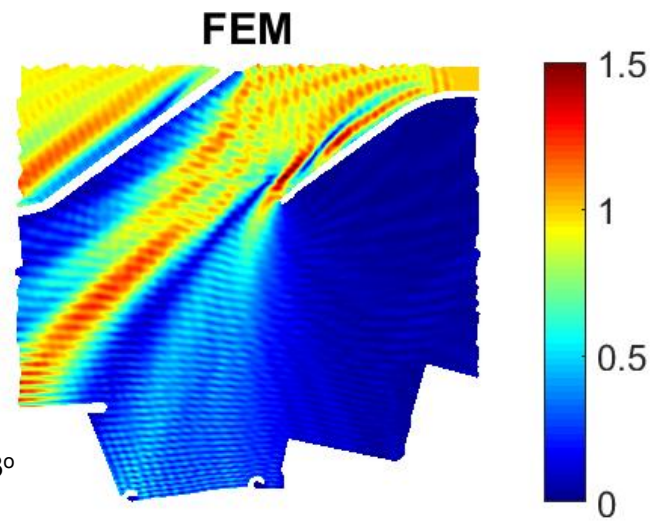

FEM

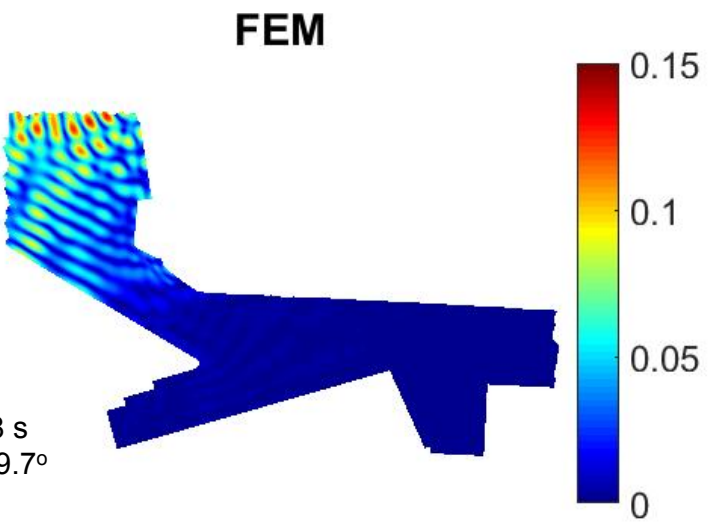

\section{FEM}

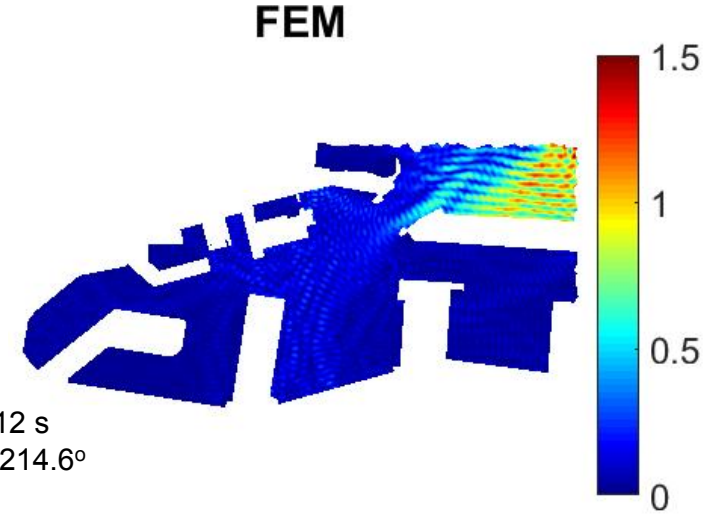

Figure 15: Barcelona harbor: wave amplification comparison between high order FEM (right column) and PGD solution (left column) for three different input parameters and areas of the harbor. 


\section{References}

[1] P. Kumar, H. Zhang, K. I. Kim, Y. Shi, and D. A. Yuen. Wave spectral modeling of multidirectional random waves in a harbor through combination of boundary integral of Helmholtz equation with Chebyshev point discretization. Comput. Fluids, 108:13$24,2015$.

[2] P. Kumar, H. Zhang, K. I. k Kim, and D. A. Yuen. Modeling wave and spectral characteristics of moored ship motion in Pohang New Harbor under the resonance conditions. Ocean Eng., 119:101-113, 2016.

[3] Giorgio Bellotti. Transient response of harbours to long waves under resonance conditions. Coast. Eng., 54:680-693, 2007.

[4] Jiin-Jen Lee, Ching-Piau Lai, and Yigong Li. Application of computer modeling for harbor resonance studies of Long Beach and Los Angeles harbor basins. In Proc. 26th Coastal Engineering Conference, volume 2, pages 1196-1209, Copenhagen, Denmark, 1998.

[5] F. Chinesta, A. Leygue, F. Bordeu, J.V. Aguado, E. Cueto, D. González, I. Alfaro, A. Ammar, and A. Huerta. PGD-Based Computational Vademecum for Efficient Design, Optimization and Control. Arch. Comput. Methods Eng., 20(1):31-59, 2013.

[6] D. Modesto, S. Zlotnik, and A. Huerta. Proper generalized decomposition for parameterized Helmholtz problems in heterogeneous and unbounded domains: Application to harbor agitation. Comput. Methods Appl. Mech. Eng., 295:127-149, 2015.

[7] A. Ammar, B. Mokdad, F. Chinesta, and R. Keunings. A new family of solvers for some classes of multidimensional partial differential equations encountered in kinetic theory modelling of complex fluids. J. Non-Newtonian Fluid Mech., 139:153-176, 2006.

[8] A. Ammar, B. Mokdad, F. Chinesta, and R. Keunings. A new family of solvers for some classes of multidimensional partial differential equations encountered in kinetic theory modeling of complex fluids. Part II: transient simulation using space-time separated representations. J. Non-Newtonian Fluid Mech., 144:98-121, 2007.

[9] F. Chinesta, P. Ladevèze, and E. Cueto. A short review on model order reduction based on proper generalized decomposition. Arch. Comput. Methods Eng., 18(4):395404, 2011.

[10] F. Chinesta, A. Ammar, and E. Cueto. Recent Advances and New Challenges in the Use of the Proper Generalized Decomposition for Solving Multidimensional Models. Arch. Comput. Methods Eng., 17(4):327-350, 2010. 
[11] S. Zlotnik, P. Díez, D. Modesto, and A. Huerta. Proper generalized decomposition of a geometrically parametrized heat problem with geophysical applications. Int. J. Numer. Methods Eng., 103(10):737-758, 2015.

[12] A. Nouy. A priori model reduction through proper generalized decomposition for solving time-dependent partial differential equations. Comput. Methods Appl. Mech. Eng., 199:1603-1626, 2010.

[13] R. Sevilla, S. Fernández-Méndez, and A. Huerta. NURBS-Enhanced Finite Element Method (NEFEM). Int. J. Numer. Methods Eng., 76(1):56-83, 2008.

[14] P. Kumar, H. Zhang, D. A. Yuen, and K. I. Kim. Wave field analysis in a harbor with irregular geometry through boundary integral of Helmholtz equation with corner contributions. Comput. Fluids, 88:287-297, 2013.

[15] G. Bellotti, R. Briganti, G. M. Beltrami, and L. Franco. Modal analysis of semienclosed basins. Coast. Eng., 64:16-25, 2012.

[16] X. Xing, Z. Kou, Z. Huang, and J.-J. Lee. Frequency Domain Response at Pacific Coast Harbors to Major Tsunamis of 2005-2011. Pure Appl. Geophys., 170(6-8):11491168, 2013.

[17] M. Guerrini, G. Bellotti, Y. Fan, and L. Franco. Numerical modelling of long waves amplification at Marina di Carrara Harbour. Appl. Ocean Res., 48:322-330, 2014.

[18] T. J. R. Hughes, J. A. Cottrell, and Y. Bazilevs. Isogeometric analysis: CAD, finite elements, NURBS, exact geometry and mesh refinement. Comput. Methods Appl. Mech. Eng., 194(39-41):4135-4195, 2005.

[19] R. Sevilla, S. Fernández-Méndez, and A. Huerta. NURBS-Enhanced Finite Element Method (NEFEM) a seamless bridge between CAD and FEM. Arch. Comput. Methods Eng., 18(4):441-484, 2011.

[20] R. Sevilla and S. Fernández-Méndez. Numerical integration over 2D NURBS-shaped domains with applications to NURBS-enhanced FEM. Finite Elem. Anal. Des., 47(10):1209-1220, 2011.

[21] R. Sevilla, S. Fernández-Méndez, and A. Huerta. NURBS-enhanced finite element method for Euler equations. Int. J. Numer. Methods Fluids, 57(9):1051-1069, 2008.

[22] R. Sevilla and A. Huerta. HDG-NEFEM with Degree Adaptivity for Stokes Flows. J. Sci. Comput., pages 1-28, 2018.

[23] J .C. W. Berkhoff. Computation of combined refraction-diffraction. In Proc. 13th Coastal Engineering Conference, volume 1, pages 471-490, Vancouver, Canada, 1972. 
[24] M. W. Dingemans. Water wave propagation over uneven bottoms, volume 13 of Advanced Series on Ocean Engineering. World Scientific, 1997.

[25] J.-P. Berenger. A perfectly matched layer for the absorption of electromagnetic waves. J. Comput. Phys., 114(2):185-200, 1994.

[26] David Modesto, Sonia Fernández-Méndez, and Antonio Huerta. Elliptic Harbor Wave Model with Perfectly Matched Layer and Exterior Bathymetry Effects. J. Waterw. Port Coast. Ocean Eng., 142(5):04016008, 2016.

[27] N. Booij. Gravity waves on water with non-uniform depth and current. Technical Report 81-1, Department of Civil Engineering, Delft University of Technology, The Netherlands, 1981.

[28] James T. Kirby. Note on linear surface wave-current interaction over slowly varying topography. J. Geophys. Res., 89(NC1):745-747, 1984.

[29] S. R. Massel. Extended refraction-diffraction equation for surface waves. Coast. Eng., 19(1-2):97-126, 1993.

[30] A. Oskooi and S. G. Johnson. Distinguishing correct from incorrect PML proposals and a corrected unsplit PML for anisotropic, dispersive media. J. Comput. Phys., 230(7):2369-2377, 2011.

[31] Ardavan F. Oskooi, Lei Zhang, Yehuda Avniel, and Steven G. Johnson. The failure of perfectly matched layers, and towards their redemption by adiabatic absorbers. Opt. Exp., 16(15):11376-11392, 2008.

[32] V. Panchang, W. Chen, B. Xu, K. Schlenker, Z. Demirbilek, and M. Okihiro. Exterior bathymetric effects in elliptic harbor wave models. J. Waterw. Port Coast. Ocean Eng., 126(2):71-78, 2000.

[33] I. Singer and E. Turkel. A perfectly matched layer for the Helmholtz equation in a semi-infinite strip. J. Comput. Phys., 201(2):439-465, 2004.

[34] A. Bermúdez, L. Hervella-Nieto, A. Prieto, and R. Rodríguez. An exact bounded perfectly matched layer for time-harmonic scattering problems. SIAM J. Sci. Comput., 30(1):312-338, 2007.

[35] T.-K. Tsay and P. L.-F. Liu. A finite element model for wave refraction and diffraction. Appl. Ocean Res., 5(1):30-37, 1983.

[36] Giorgio Giorgiani, David Modesto, Sonia Fernández-Méndez, and Antonio Huerta. High-order continuous and discontinuous Galerkin methods for wave problems. Int. J. Numer. Methods Fluids, 73(10):883-903, 2013. 
[37] R. Sevilla, S. Fernández-Méndez, and A. Huerta. 3D NURBS-enhanced finite element method (NEFEM). Int. J. Numer. Methods Eng., 88(2):103-125, 2011.

[38] R. Sevilla, L. Rees, and O. Hassan. The generation of triangular meshes for NURBSenhanced FEM. Int. J. Numer. Methods Eng., 108(8):941-968, 2016.

[39] C. Bunks, F. M. Saleck, S. Zaleski, and G. Chavent. Multiscale seismic waveform inversion. Geophysics, 60(5):1457-1473, 1995.

[40] A. Badías, D. González, I. Alfaro, F. Chinesta, and E. Cueto. Local proper generalized decomposition. International Journal for Numerical Methods in Engineering, 112(12):1715-1732, 2017.

[41] A. Ammar, F. Chinesta, P. Díez, and A. Huerta. An error estimator for separated representations of highly multidimensional models. Comput. Methods Appl. Mech. Eng., 199:1872-1880, 2010. 